\title{
Threshold solutions for the focusing 3D cubic Schrödinger equation
}

\section{Thomas Duyckaerts and Svetlana Roudenko}

\begin{abstract}
We study the focusing $3 \mathrm{~d}$ cubic NLS equation with $H^{1}$ data at the mass-energy threshold, namely, when $M\left[u_{0}\right] E\left[u_{0}\right]=M[Q] E[Q]$. In earlier works of Holmer-Roudenko and Duyckaerts-Holmer-Roudenko, the behavior of solutions (i.e., scattering and blow up in finite time) was classified when $M\left[u_{0}\right] E\left[u_{0}\right]<M[Q] E[Q]$. In this paper, we first exhibit 3 special solutions: $e^{i t} Q$ and $Q^{ \pm}$, where $Q$ is the ground state, $Q^{ \pm}$exponentially approach the ground state solution in the positive time direction, $Q^{+}$has finite time blow up and $Q^{-}$scatters in the negative time direction. Secondly, we classify solutions at this threshold and obtain that up to $\dot{H}^{1 / 2}$ symmetries, they behave exactly as the above three special solutions, or scatter and blow up in both time directions as the solutions below the mass-energy threshold. These results are obtained by studying the spectral properties of the linearized Schrödinger operator in this mass-supercritical case, establishing relevant modulational stability and careful analysis of the exponentially decaying solutions to the linearized equation.
\end{abstract}

\section{Introduction}

We consider the 3d focusing cubic nonlinear Schrödinger (NLS) equation on a time interval $I \subset \mathbb{R}(0 \in I)$

$$
\left\{\begin{array}{c}
i \partial_{t} u+\Delta u+|u|^{2} u=0, \quad(x, t) \in \mathbb{R}^{3} \times I \\
u_{\mid t=0}=u_{0} \in H^{1}\left(\mathbb{R}^{3}\right) .
\end{array}\right.
$$

2000 Mathematics Subject Classification: 35Q55, 35P25, 35B40.

Keywords: Nonlinear Schrödinger equation, scattering, profile decomposition, blow-up. 
The Cauchy problem (1.1) is locally wellposed in $H^{1}$, see [12]. We denote the forward lifespan by $\left[0, T_{+}\right)$and the backward by $\left(T_{-}, 0\right]$. If $T_{+}(u)<+\infty$ [or $\left.T_{-}(u)>-\infty\right]$, then $\|u(t)\|_{H^{1}}$ tends to $+\infty$ as $t$ tends to $T_{+}$[respectively, $t$ tends to $T_{-}$] and it is said that the solution blows up in finite time.

The solutions of (1.1) satisfy mass, energy and momentum conservation laws

$$
\begin{aligned}
& E[u](t)=\frac{1}{2} \int|\nabla u(x, t)|^{2} d x-\frac{1}{4} \int|u(x, t)|^{4} d x=E[u](0), \\
& M[u](t)=\int|u(x, t)|^{2} d x=M[u](0) \\
& P[u](t)=\operatorname{Im} \int \bar{u}(x, t) \nabla u(x, t) d x=P[u](0) .
\end{aligned}
$$

Furthermore, this NLS equation enjoys several invariances. If $u(t, x)$ is a solution, then

- by scaling invariance: so is $\lambda u\left(\lambda x, \lambda^{2} t\right), \lambda>0$;

- by spatial translation: so is $u\left(x+x_{0}, t\right)$ for $x_{0} \in \mathbb{R}^{3}$;

- by time translation: so is $u\left(x, t+t_{0}\right)$ for $t_{0} \in \mathbb{R}$;

- by phase rotation invariance: so is $e^{i \theta_{0}} u, \theta_{0} \in \mathbb{R}$;

- by time reversal symmetry: so is $\overline{u(x,-t)}$.

Observe that all these transformations leave the $\dot{H}^{1 / 2}$-norm and the momentum invariant. In what follows the solutions will be considered up to the $\left(\dot{H}^{1 / 2}-\right)$ symmetries of this NLS equation meaning up to the above mentioned invariances.

A transformation of solutions to (1.1), which does not leave the $\dot{H}^{1 / 2}$-norm nor the momentum invariant, is the Galilean transformation: if $u$ is a solution, so is

$$
e^{i x \xi_{0}} e^{-i t\left|\xi_{0}\right|^{2}} u\left(x-2 \xi_{0} t, t\right), \xi_{0} \in \mathbb{R}^{3} .
$$

Consider a general focusing NLS equation

$$
i \partial_{t} u+\Delta u+|u|^{p-1} u=0, \quad(x, t) \in \mathbb{R}^{d} \times \mathbb{R},
$$

with the nonlinearity $p>1$ and the dimension $d$ such that $0 \leq s_{c} \leq 1$, where $s_{c}=\frac{d}{2}-\frac{2}{p-1}$. The case when $s_{c}=0$ is referred to as mass (or $L^{2}$ )-critical, the case when $s_{c}=1$ is called energy-critical, and in our case, the NLS equation in (1.1) has $s_{c}=1 / 2$, and thus, is referred to as $\dot{H}^{1 / 2}$-critical. 
The focusing mass-critical NLS equation (for example, cubic NLS in 2d) with $H^{1}$ initial data was originally studied by Weinstein [32], who showed that there exists a sharp threshold, which splits the behavior of solutions: (i) if $M[u]<M[Q]$, then the solution exists globally in time and (ii) if $M[u] \geq M[Q]$, then the solution may blow up in finite time. Here, $Q$ is the ground state solution of $-Q+\Delta Q+|Q|^{4 / d} Q=0, Q=Q(r), r=|x|$, $x \in \mathbb{R}^{d}$. In the first case the scattering was known for the initial data in $L^{2}$ and of finite variance (it follows from the pseudoconformal conservation law, e.g., see [32], [3]). The scattering for radially symmetric solutions with $L^{2}$ initial data was recently established in [20] for $2 \mathrm{~d}$, and in [22] for higher dimensions. For general $L^{2}$ initial data scattering is still an open question.

Note that the solution $u(x, t)=e^{i t} Q(x)$ (it has $M[u]=M[Q]$ ) exists globally in time (in fact, it is time-periodic), but does not scatter. Under the pseudoconformal transformation this solution can be mapped into a finite time blow up solution (of the same mass). Merle has shown that all finite time blow up solutions of minimal mass $M[u]=M[Q]$ are pseudoconformal images (up to phase, translation, scaling and Galilean invariances) of $e^{i t} Q(x)$, see [26] for radial $H^{1}$ data with finite variance and [27] for general $H^{1}$ data. Furthermore, he characterized all $H^{1}$ solutions of finite variance with the threshold mass $M[u]=M[Q]$ : a solution can be a scaled version of the time periodic solution $e^{i t} Q(x)$, or a blow up solution which is a pseudoconformal image of $e^{i t} Q(x)$ (a "self-similar solution"), or a globally defined solution with quadratically decaying in time $L^{4 / d+2}$ norm which implies scattering as $t \rightarrow \pm \infty$.

The focusing energy critical NLS equation (for example, cubic NLS in 4d or quintic NLS in 3d) was recently studied by Kenig-Merle [16]. They showed that (in dimensions 3,4 and 5) a sharp splitting takes place for the Cauchy problem with $\dot{H}_{\text {rad }}^{1}$ initial data and an a priori condition $E\left[u_{0}\right]<E[W]$ : (i) if $\left\|\nabla u_{0}\right\|_{L^{2}}<\|\nabla W\|_{L^{2}}$, then the solution exists globally in time, moreover, it scatters; (ii) if $\left\|\nabla u_{0}\right\|_{L^{2}}>\|\nabla W\|_{L^{2}}$, and $u_{0} \in L^{2}$, then finite time blow up occurs $^{1}$. Here, $W$ is the stationary solution of (1.1) in $\dot{H}^{1}$, given explicitly by $W(r)=1 /\left(1+\frac{r^{2}}{d(d-2)}\right)^{(d-2) / 2}, r=|x|, x \in \mathbb{R}^{d}$. A similar result (but not necessarily for radial initial data) is established by the same authors for the energy-critical focusing nonlinear wave (NLW) equation in [17].

Observe that the above characterization is obtained only if $E\left[u_{0}\right]<$ $E[W]$. What happens if this condition is removed? The case of the critical level of energy, i.e., $E\left[u_{0}\right]=E[W]$, was recently studied by Duyckaerts-Merle in [8]. Richer dynamics for the behavior of solutions as $t \rightarrow \pm \infty$ are exhibited. Besides the stationary solution $W$ which exists globally but does not

\footnotetext{
${ }^{1}$ Under the above a priori condition the gradients of $u_{0}$ and $W$ can not be equal.
} 
scatter, there are two more special solutions $W^{-}$and $W^{+}$which approach $W$ in $\dot{H}^{1}$ in one time direction, but in the opposite time direction $W^{-}$scatters and $W^{+}$blows up in finite time ${ }^{2}$. The deciding factor is the gradient size: $\left\|\nabla W^{-}\right\|_{L^{2}}<\|\nabla W\|_{L^{2}}$ and $\left\|\nabla W^{+}\right\|_{L^{2}}>\|\nabla W\|_{L^{2}}$. Moreover, the classification of all (radial) solutions at the energy critical level is given (up to the symmetries of the equation): if $\left\|\nabla u_{0}\right\|_{L^{2}}<\|\nabla W\|_{L^{2}}$, then the solution is $W^{-}$, if $\left\|\nabla u_{0}\right\|_{L^{2}}>\|\nabla W\|_{L^{2}}$ (with the additional technical assumption that $u_{0}$ is in $L^{2}$ ), then the solution is $W^{+}$, and when $\left\|\nabla u_{0}\right\|_{L^{2}}=\|\nabla W\|_{L^{2}}$, then the solution is $W$. A similar result is obtained for the energy-critical focusing NLW equation for general initial data in Duyckaerts-Merle [9].

The results on global existence or finite time blow up for the mass-critical NLS and energy-critical NLS equations can be linked by studying the NLS equation with $0<s_{c}<1$ considered in Holmer-Roudenko [14, Section 2], see also [15, Section 7]. For the purpose of this paper we will state the result only for the physical cubic equation (1.1), i.e., when $s_{c}=1 / 2$. The scattering result in the following theorem was established initially for the radial $H^{1}$ data in [15] and the radiality assumption was removed in [7].

Let $Q$ be the ground state, that is the unique positive radial solution of the equation $-Q+\Delta Q+|Q|^{2} Q=0$ (see Subsection 2.1 for the details).

Theorem 1 ([14, 15, 7]). Let $u$ be an $H^{1}$ solution to (1.1). Suppose

$$
M\left[u_{0}\right] E\left[u_{0}\right]<M[Q] E[Q] .
$$

- If $\left\|u_{0}\right\|_{L^{2}}\left\|\nabla u_{0}\right\|_{L^{2}}<\|Q\|_{L^{2}}\|\nabla Q\|_{L^{2}}$, then

$$
\left\|u_{0}\right\|_{L^{2}}\|\nabla u(t)\|_{L^{2}}<\|Q\|_{L^{2}}\|\nabla Q\|_{L^{2}}
$$

for all $t$ in the interval of existence of $u$, and thus, the solution $u$ is globally defined; moreover, it scatters in $H^{1}$.

- If $\left\|u_{0}\right\|_{L^{2}}\left\|\nabla u_{0}\right\|_{L^{2}}>\|Q\|_{L^{2}}\|\nabla Q\|_{L^{2}}$, then

$$
\left\|u_{0}\right\|_{L^{2}}\|\nabla u(t)\|_{L^{2}}>\|Q\|_{L^{2}}\|\nabla Q\|_{L^{2}}
$$

for all $t$ in the interval of existence of $u$, and, if either $u_{0}$ is radial or has a finite variance, i.e., $|x| u_{0} \in L^{2}$, then the solution $u$ blows up in finite time.

As in the preceding cases, the determining quantities

$$
M[u] E[u] \text { and }\left\|u_{0}\right\|_{L^{2}}\left\|\nabla u_{0}\right\|_{L^{2}}
$$

are invariant by the scaling of the equation.

\footnotetext{
${ }^{2}$ The blow up is shown for $d=5$ and conjectured for other dimensions.
} 
Techniques employed above are based on the approach of Kenig-Merle in [16] and [17]. In particular, scattering is established by the profile decomposition method by Gérard [10], which is a refinement of the concentrationcompactness method of P.-L. Lions [24, 25]; see previous applications to NLS by Merle-Vega [29] and Keraani [19], and to NLW equation by BahouriGérard [1]. For other recent applications of profile decomposition we refer the reader to the works of Gérard [11] on the 3d cubic wave equation, and of Kenig-Merle [18], who established scattering of solutions for the defocusing cubic NLS in 3d (equation (1.1) with a minus sign in front of the nonlinearity) with $\dot{H}^{1 / 2}$ initial data provided $\dot{H}^{1 / 2}$ norm stays bounded globally in time (see also Colliander-Keel-Staffilani-Takaoka-Tao [6] and references therein for previous results on defocusing problems).

Coming back to Theorem 1, we would like to describe the behavior of solutions to (1.1) at the "critical" mass-energy threshold, i.e., when

$$
M[u] E[u]=M[Q] E[Q] .
$$

First, we establish the existence of special solutions (besides $e^{i t} Q$ ) at the critical mass-energy threshold.

Theorem 2. There exist two radial solutions $Q^{+}$and $Q^{-}$of (1.1) with initial conditions $Q_{0}^{ \pm}$such that $Q_{0}^{ \pm} \in \cap_{s \in \mathbb{R}} H^{s}\left(\mathbb{R}^{3}\right)$ and

a. $M\left[Q^{+}\right]=M\left[Q^{-}\right]=M[Q], E\left[Q^{+}\right]=E\left[Q^{-}\right]=E[Q],[0,+\infty)$ is in the (time) domain of definition of $Q^{ \pm}$and there exists $e_{0}>0$ such that

$$
\forall t \geq 0, \quad\left\|Q^{ \pm}(t)-e^{i t} Q\right\|_{H^{1}} \leq C e^{-e_{0} t}
$$

b. $\left\|\nabla Q_{0}^{-}\right\|_{2}<\|\nabla Q\|_{2}, Q^{-}$is globally defined and scatters for negative time,

c. $\left\|\nabla Q_{0}^{+}\right\|_{2}>\|\nabla Q\|_{2}$, and the negative time of existence of $Q^{+}$is finite.

Remark. The best constant $-e_{0}$ in (a) is given by the negative eigenvalue of the linearized operator associated to (1.1) around the periodic solution $e^{i t} Q$. Furthermore, the construction of $Q^{ \pm}$gives an asymptotic expansion in all Sobolev spaces for all orders of $e^{-e_{0} t}$ of $Q^{ \pm}$as $t \rightarrow+\infty$. Such precise information is not available for negative times. In particular, we are not able to describe the behavior of $Q^{+}$near the blow-up time, except for what is already known for general blow-up solutions of (1.1) (see [28], [14]). follows:

Next, we characterize all solutions at the critical mass-energy level as 
Theorem 3. Let $u$ be a solution of (1.1) satisfying (1.3).

a. If $\left\|\nabla u_{0}\right\|_{2}\left\|u_{0}\right\|_{2}<\|\nabla Q\|_{2}\|Q\|_{2}$, then either $u$ scatters or $u=Q^{-}$up to the symmetries.

b. If $\left\|\nabla u_{0}\right\|_{2}\left\|u_{0}\right\|_{2}=\|\nabla Q\|_{2}\|Q\|_{2}$, then $u=e^{i t} Q$ up to the symmetries.

c. If $\left\|\nabla u_{0}\right\|_{2}\left\|u_{0}\right\|_{2}>\|\nabla Q\|_{2}\|Q\|_{2}$ and $u_{0}$ is radial or of finite variance, then either the interval of existence of $u$ is of finite length or $u=Q^{+}$ up to the symmetries.

Note that as a consequence of (1.3), the assumptions $\left\|\nabla u\left(t_{0}\right)\right\|_{2}\left\|u\left(t_{0}\right)\right\|_{2}<$ $\|\nabla Q\|_{2}\|Q\|_{2}$ and $\left\|\nabla u\left(t_{0}\right)\right\|_{2}\left\|u\left(t_{0}\right)\right\|_{2}>\|\nabla Q\|_{2}\|Q\|_{2}$ do not depend on the choice of the initial condition (see $\S 2.3$ ).

Remark. It is worth linking the $\dot{H}^{1 / 2}$-critical equation (1.1) with the corresponding mass-critical and energy-critical once again. The proofs of Theorems 2 and 3 will show that the behavior of the solutions of (1.1) at the threshold is very close to the one of the energy-critical equation described in the radial case in [8]. In particular, in both cases, the existence of the special solutions $Q^{ \pm}\left(W^{ \pm}\right.$in the energy-critical case) derives from the existence of two real nonzero eigenvalues for the linearized operator around the periodic solution $e^{i t} Q$ (respectively around the stationary solution $W$ ). On the other hand, in the mass-critical case, the only eigenvalue of the linearized operator is 0 (see [33]), and the blow-up solution at the threshold is given by the pseudo-conformal transformation, which is specific to the mass-critical equation.

Note that results in this paper are more complete than those in [8], which are restricted to radial solutions ${ }^{3}$ and the blow-up of the special solution $W^{+}$ is shown only in the space dimension 5. This is due to the fact that we have more freedom in our setting than in the energy-critical one: the set of solutions of (1.1) is stable by the Galilean transformation and the ground state $Q$ decays exponentially at infinity.

We next give a formulation of Theorems 1 and 3 that takes the Galilean transformation into account. Let $u$ be a solution of (1.1). Applying to $u$, as in $\left[7\right.$, Section 4], the Galilean transformation with parameter $\xi_{0}=-P[u] / M[u]$, we get a solution $v$ of (1.1) with zero momentum which is the minimal energy solution among all Galilean transformations of $u$. Precisely,

$$
M[v]=M[u], \quad E[v]=E[u]-\frac{1}{2} \frac{P[u]^{2}}{M[u]}, \quad\left\|\nabla v_{0}\right\|_{L^{2}}^{2}=\left\|\nabla u_{0}\right\|_{L^{2}}^{2}-\frac{P\left[u_{0}\right]^{2}}{M\left[u_{0}\right]} .
$$

\footnotetext{
${ }^{3}$ See [21] for a nonradial generalization of [16] in dimension 5 and higher that should extend to the energy threshold.
} 
Applying Theorems 1 and 3 to $v$, we get

Theorem 4. Let $u$ be a solution of (1.1) satisfying

$$
E[u] M[u]-\frac{1}{2} P[u]^{2} \leq E[Q] M[Q] .
$$

Then

a. If $\left\|\nabla u_{0}\right\|_{2}^{2}\left\|u_{0}\right\|_{2}^{2}-P[u]^{2}<\|\nabla Q\|_{2}^{2}\|Q\|_{2}^{2}$, then either $u$ scatters or $u=Q^{-}$ up to the symmetries.

b. If $\left\|\nabla u_{0}\right\|_{2}^{2}\left\|u_{0}\right\|_{2}^{2}-P[u]^{2}=\|\nabla Q\|_{2}^{2}\|Q\|_{2}^{2}$, then $u=e^{i t} Q$ up to the symmetries.

c. If $\left\|\nabla u_{0}\right\|_{2}^{2}\left\|u_{0}\right\|_{2}^{2}-P[u]^{2}>\|\nabla Q\|_{2}^{2}\|Q\|_{2}^{2}$ and $u_{0}$ is radial or of finite variance, then either the interval of existence of $u$ is of finite length or $u=Q^{+}$up to the symmetries.

In the preceding theorem, up to the symmetries of the equation means up to the $\dot{H}^{1 / 2}$-symmetries and the Galilean transformation. If $E[u] M[u] \leq$ $E[Q] M[Q]$, our results actually show that the condition

$$
\left\|\nabla u_{0}\right\|_{2}^{2}\left\|u_{0}\right\|_{2}^{2}>\|\nabla Q\|_{2}^{2}\|Q\|_{2}^{2}
$$

implies the stronger bound

$$
\left\|\nabla u_{0}\right\|_{2}^{2}\left\|u_{0}\right\|_{2}^{2}-P\left[u_{0}\right]^{2}>\|\nabla Q\|_{2}^{2}\|Q\|_{2}^{2} .
$$

The paper is organized as follows. In the next section we recall the properties of the ground state $Q$, small data theory for Cauchy problem (1.1) and the spectral properties of the linearized (around the ground state solution $e^{i t} Q$ ) Schrödinger operator. Under the condition (1.3) we identify a quadratic form associated to the linearized Schrödinger operator which can measure closeness to $e^{i t} Q$ and find subspaces of $H^{1}$ where this form is positive, avoiding thus vanishing and negative directions.

In Section 3 we construct a family of approximate solutions using the knowledge about the discrete spectrum of the linearized operator and then with a fixed point argument produce candidates for the special solutions $Q^{-}$ and $Q^{+}$, thus, proving Theorem 2 except for the negative time behavior of $Q^{ \pm}$.

In Section 4 we discuss the modulational stability near the ground state solution. Here, we identify the spatial and phase parameters which control the variations from $e^{i t} Q$ (on the subsets where the above mentioned quadratic form is positive) while the entire variation being small in $H^{1}$ norm. 
In Sections 5 and 6 we study solutions with initial data from Theorem 3 part (a) and (c), respectively. Our main goal is to obtain exponential convergence for large (positive) time of the gradient variation (4.16) which then will imply exponential convergence in (positive) time to $e^{i t} Q$ (up to spatial translation and phase rotation), see Lemma 4.4. We also finish Theorem 2 for negative time behavior.

In Section 7 we first analyze exponentially small solutions of the linearized Schrödinger equation and then establish the uniqueness of special solutions. We finish the section with the classification of solutions result.

Appendix contains the proof of coercivity of the quadratic form introduced in Section 3 where we follow Weinstein [33] and a useful inequality, the original idea of which is due to Banica [2].

\section{Notation}

Let $\mathcal{S}$ denote the space of Schwartz functions, i.e, the topological space of functions $v$ satisfying

$$
\forall \alpha, N, \quad\|v\|_{\alpha, N}:=\sup _{x \in \mathbb{R}^{3}}\left|(1+|x|)^{N} \partial_{x}^{\alpha} v(x)\right|<\infty,
$$

with the topology given by the family of semi-norms $\|\cdot\|_{\alpha, N}$.

By $H^{s}$ we denote the usual Sobolev space of smoothness $s$ in spatial (on $\mathbb{R}^{3}$ ) variable. Let $H^{\infty}=\bigcap_{s \in \mathbb{R}} H^{s}$. We denote by $\|\cdot\|_{p}$ the $L^{p}$-norm in spatial variable.

A pair $(q, r)$ is $\dot{H}^{s}$-admissible when $\frac{2}{q}+\frac{3}{r}=\frac{3}{2}-s$ and $2 \leq q, r \leq \infty$. Consider the following Strichartz norm for functions $u$ of space and time

$$
\|u\|_{S\left(\dot{H}^{1 / 2}\right)}=\sup _{\substack{(q, r) \dot{H}^{1 / 2}-\text { admissible } \\ 4^{+} \leq q \leq \infty, 3 \leq r \leq 6^{-}}}\|u\|_{L_{t}^{q}, L_{x}^{r}},
$$

where $6^{-}<6$ (respectively $4^{+}>4$ ) is an arbitrary fixed number, close to 6 (respectively, to 4). We will also write, if $I$ is an interval and $\chi_{I}$ is the characteristic function of $I$,

$$
\|u\|_{S\left(I ; \dot{H}^{1 / 2}\right)}=\left\|\chi_{I} u\right\|_{S\left(\dot{H}^{1 / 2}\right)} .
$$

If $a$ and $b$ are two positive functions of $t$, we will write $a=O(b)$ when there exists a constant $C>0$ (independent of $t$ ) such that $a(t) \leq C b(t)$, for all $t$, and $a \approx b$ when $a=O(b)$ and $b=O(a)$.

Throughout the paper, $C$ denotes a large positive constant and $c$ a small positive constant, that do not depend on the parameters and may change from line to line. 


\section{Preliminaries}

\subsection{Properties of the ground state}

We recall some well-known properties of the ground state and refer the reader to [5], [32], [23] as well as [4], [31, Appendix B], [15, §3] for more details.

Consider the nonlinear elliptic equation

$$
-Q+\Delta Q+|Q|^{2} Q=0 .
$$

The $H^{1}$ solutions of this equation can be enumerated by their mass $\left(L^{2}\right.$ norm) and the minimal mass solution, $Q$, is called the ground state. The function $Q$ is radial, smooth, positive, exponentially decaying at infinity, and characterized as the unique minimizer for the Gagliardo-Nirenberg inequality: if $u \in H^{1}\left(\mathbb{R}^{3}\right)$,

$$
\|u\|_{4}^{4} \leq C_{G N}\|\nabla u\|_{2}^{3}\|u\|_{2}, \quad\|Q\|_{4}^{4}=C_{G N}\|\nabla Q\|_{2}^{3}\|Q\|_{2},
$$

and

(2.3) $\|u\|_{4}^{4}=C_{G N}\|\nabla u\|_{2}^{3}\|u\|_{2} \Longrightarrow \exists \lambda_{0} \in \mathbb{C}, \exists x_{0} \in \mathbb{R}^{3}: u(x)=\lambda_{0} Q\left(x+x_{0}\right)$.

The above characterization of $Q$ and the concentration-compactness principle (see [24, Theorem I.2]) yield:

Proposition 2.1. There exists a function $\varepsilon(\rho)$, defined for small $\rho>0$, such that $\lim _{\rho \rightarrow 0} \varepsilon(\rho)=0$ and

$$
\begin{aligned}
& \forall u \in H^{1}, \quad \mid\|u\|_{4}-\|Q\|_{4}|+|\|u\|_{2}-\|Q\|_{2}|+|\|\nabla u\|_{2}-\|\nabla Q\|_{2} \mid \leq \rho \\
& \Longrightarrow \exists \theta_{0} \in \mathbb{R}, x_{0} \in \mathbb{R}^{3}, \quad\left\|u-e^{i \theta_{0}} Q\left(\cdot-x_{0}\right)\right\|_{H^{1}} \leq \varepsilon(\rho) .
\end{aligned}
$$

We will make the statement of Proposition 2.1 more precise in $\S 4$.

We will also need the following equalities, consequences of Pohozhaev identities (see e.g $[15, \S 3]$ ):

$$
\|Q\|_{4}^{4}=4\|Q\|_{2}^{2} \quad \text { and } \quad\|\nabla Q\|_{2}^{2}=3\|Q\|_{2}^{2} .
$$

\subsection{The Cauchy problem (1.1)}

Here, we briefly recall global existence and scattering results for (1.1), for more details see [15]. The small data theory states that there exists a small $\epsilon_{s d}>0$ such that if

$$
\left\|e^{i t \triangle} u_{0}\right\|_{S\left([0,+\infty), \dot{H}^{1 / 2}\right)} \leq \epsilon_{s d},
$$

then the solution $u$ of $(1.1)$ has $T_{+}\left(u_{0}\right)=+\infty$ and

$$
\exists C>0:\|u\|_{S\left([0,+\infty) ; \dot{H}^{1 / 2}\right)} \leq C\left\|e^{i t \triangle} u_{0}\right\|_{S\left([0,+\infty) ; \dot{H}^{1 / 2}\right)} .
$$


Next, if $u(t)$ is a solution which is globally defined for positive time, then it scatters in $H^{1}$ as $t \rightarrow+\infty$, meaning that for some $\phi \in H^{1}$,

$$
\lim _{t \rightarrow+\infty}\left\|u(t)-e^{i t \triangle} \phi\right\|_{H^{1}}=0
$$

if it has a uniformly bounded $H^{1}$ norm for $t \geq 0$ and a finite Strichartz $S\left([0,+\infty) ; \dot{H}^{1 / 2}\right)$ norm. Similar statements hold for negative times.

\subsection{Gradient separation}

Lemma 2.2. Consider (1.1) and suppose (1.3) holds.

a. If $\left\|u_{0}\right\|_{2}\left\|\nabla u_{0}\right\|_{2}=\|Q\|_{2}\|\nabla Q\|_{2}$, then $u=e^{i t} Q$ up to the invariance of the equation.

b. If $\left\|u_{0}\right\|_{2}\left\|\nabla u_{0}\right\|_{2}<\|Q\|_{2}\|\nabla Q\|_{2}$, then $u$ is globally defined and

$$
\left\|u_{0}\right\|_{2}\|\nabla u(t)\|_{2}<\|Q\|_{2}\|\nabla Q\|_{2} \text { for all } t \text {. }
$$

c. If $\left\|u_{0}\right\|_{2}\left\|\nabla u_{0}\right\|_{2}>\|Q\|_{2}\|\nabla Q\|_{2}$, then $\left\|u_{0}\right\|_{2}\|\nabla u(t)\|_{2}>\|Q\|_{2}\|\nabla Q\|_{2}$ for all $t$ in the domain of existence of $u$.

Proof. Without loss of generality we can assume $M[u]=M[Q]$ and $E[u]=$ $E[Q]$ due to scaling: if $M[u]=\alpha M[Q]$ for some $\alpha>0$, then define $\tilde{u}(x, t)=$ $\alpha u\left(\alpha x, \alpha^{2} t\right)$ which is also a solution of (1.1), and observe that

$$
M[\tilde{u}]=\frac{1}{\alpha} M[u]=M[Q]
$$

and also

$$
E[\tilde{u}]=\alpha E[u]=\alpha \frac{M[Q] E[Q]}{M[u]}=E[Q] .
$$

Case (a) is given by the variational characterization (2.3) of $Q$ and the uniqueness of solutions of (1.1).

For case (b) we show that if $\|\nabla u(t)\|_{2}<\|\nabla Q\|_{2}$ holds for $t=0$, then it does for all $t$. To the contrary, suppose (by continuity) there exists $t_{1}$ such that $\left\|\nabla u\left(t_{1}\right)\right\|_{2}=\|\nabla Q\|_{2}$. Then by case (a) with the initial condition at $t=t_{1}$, the equality holds for all times $t$ contradicting the condition at $t=0$. Hence, such $t_{1}$ does not exist and the gradient of $u(t)$ is bounded as claimed. By the finite blow-up criterion $u$ is globally defined.

Case (c) is similar to case (b). 


\subsection{Spectral properties of the linearized operator}

Consider a solution $u$ of (1.1) close to $e^{i t} Q$ and write $u$ as

$$
u(x, t)=e^{i t}(Q(x)+h(x, t)) .
$$

Let $h_{1}=\operatorname{Re} h$ and $h_{2}=\operatorname{Im} h$. We will often identify $\mathbb{C}$ and $\mathbb{R}^{2}$ and consider $h=h_{1}+i h_{2} \in \mathbb{C}$ as an element $\left(\begin{array}{l}h_{1} \\ h_{2}\end{array}\right)$ of $\mathbb{R}^{2}$. Note that $h$ is a solution of the equation

$$
\partial_{t} h+\mathcal{L} h=R(h), \quad \mathcal{L}:=\left(\begin{array}{cc}
0 & -L_{-} \\
L_{+} & 0
\end{array}\right)
$$

where the self-adjoint operators $L_{+}$and $L_{-}$and the remainder $R(h)$ are defined by

$$
\begin{aligned}
L_{+} h_{1} & :=-\Delta h_{1}+h_{1}-3 Q^{2} h_{1}, \quad L_{-} h_{2}:=-\Delta h_{2}+h_{2}-Q^{2} h_{2}, \\
R(h) & :=i Q\left(2|h|^{2}+h^{2}\right)+i|h|^{2} h .
\end{aligned}
$$

The spectral properties of the operator $\mathcal{L}$ are well known and for the following proposition we refer to [13, Theorem 3.1 and Corollary 3.1] and [33, Proposition 2.8].

Proposition 2.3. Let $\sigma(\mathcal{L})$ be the spectrum of the operator $\mathcal{L}$, defined on $L^{2}\left(\mathbb{R}^{3}\right) \times L^{2}\left(\mathbb{R}^{3}\right)$ and let $\sigma_{\mathrm{ess}}(\mathcal{L})$ be its essential spectrum. Then

$\sigma_{\text {ess }}(\mathcal{L})=\{i \xi: \xi \in \mathbb{R},|\xi| \geq 1\}, \quad \sigma(\mathcal{L}) \cap \mathbb{R}=\left\{-e_{0}, 0,+e_{0}\right\} \quad$ with $e_{0}>0$.

Furthermore, $e_{0}$ and $-e_{0}$ are simple eigenvalues of $\mathcal{L}$ with eigenfunctions $\mathcal{Y}_{+}$ and $\mathcal{Y}_{-}=\overline{\mathcal{Y}}_{+}$in $\mathcal{S}$, and the null-space of $\mathcal{L}$ is spanned by the four vectors $\partial_{x_{j}} Q, j=1,2,3$ and $i Q$.

Remark 2.4. Let $\mathcal{Y}_{1}=\operatorname{Re} \mathcal{Y}_{+}=\operatorname{Re} \mathcal{Y}_{-}$and $\mathcal{Y}_{2}=\operatorname{Im} \mathcal{Y}_{+}=-\operatorname{Im} \mathcal{Y}_{-}$. Then

$$
L_{+} \mathcal{Y}_{1}=e_{0} \mathcal{Y}_{2} \quad \text { and } \quad L_{-} \mathcal{Y}_{2}=-e_{0} \mathcal{Y}_{1}
$$

Remark 2.5. Proposition 2.3 implies that the null-space of $L_{+}$is spanned by $\partial_{x_{1}} Q, \partial_{x_{2}} Q$ and $\partial_{x_{3}} Q$ and the null-space of $L_{-}$is spanned by $Q$.

Remark 2.6. It also follows from Proposition 2.8 of [33] that $\int\left(L_{-} f\right) f \geq 0$ for all real-valued $h$ in $H^{1}$. Together with the preceding remark, we get

$$
\forall f \in H^{1} \backslash\{\lambda Q, \lambda \in \mathbb{R}\}, \quad \int\left(L_{-} f\right) f>0 .
$$


Consider the linearized equation $\partial_{t} h+\mathcal{L} h=0$. Multiply by $\overline{i \partial_{t} h}$ and take the real part to obtain

$$
\partial_{t} \int\left(L_{+} h_{1}\right) h_{1}+\partial_{t} \int\left(L_{-} h_{2}\right) h_{2}=0
$$

Define $\Phi$, a linearized energy, by

$$
\begin{aligned}
\Phi(h) & :=\frac{1}{2} \int|h|^{2}+\frac{1}{2} \int|\nabla h|^{2}-\frac{1}{2} \int Q^{2}\left(3 h_{1}^{2}+h_{2}^{2}\right) \\
& =\frac{1}{2} \int\left(L_{+} h_{1}\right) h_{1}+\frac{1}{2} \int\left(L_{-} h_{2}\right) h_{2} .
\end{aligned}
$$

From (2.10) it follows that $\Phi$ is conserved for solutions of the linearized equation $\partial_{t} h+\mathcal{L} h=0$. By explicit calculation (see the beginning of Appendix A for the details),

$$
\begin{aligned}
E[Q+h]=E[Q] \text { and } & M[Q+h]=M[Q] \\
\Longrightarrow \Phi(h) & =\int Q|h|^{2} h_{1}+\frac{1}{4} \int|h|^{4}
\end{aligned}
$$

which shows that $|\Phi(h)| \leq c\|h\|_{4}^{3}$ for a threshold solution $u=e^{i t}(Q+h)$ of (1.1) which is close to $e^{i t} Q$. To take advantage of this, we next study the sign of $\Phi(h)$.

We denote by $B(g, h)$ the bilinear symmetric form associated to $\Phi$, i.e., for $g, h \in H^{1}\left(\mathbb{R}^{3}\right)$

$$
B(g, h)=\frac{1}{2} \int\left(L_{+} g_{1}\right) h_{1}+\frac{1}{2} \int\left(L_{-} g_{2}\right) h_{2} .
$$

By Remark 2.5,

(2.14) $\forall h \in H^{1}\left(\mathbb{R}^{3}\right), B\left(\partial_{x_{1}} Q, h\right)=B\left(\partial_{x_{2}} Q, h\right)=B\left(\partial_{x_{3}} Q, h\right)=B(i Q, h)=0$.

Furthermore, by (2.4)

$$
\Phi(Q)=\frac{1}{2} \int|Q|^{2}+\frac{1}{2} \int|\nabla Q|^{2}-\frac{3}{2} \int Q^{4}=-4 \int Q^{2}<0
$$

Together with (2.14) we get

$$
\forall h \in \operatorname{span}\left\{\partial_{x_{1}} Q, \partial_{x_{2}} Q, \partial_{x_{3}} Q, i Q, Q\right\}, \quad \Phi(h) \leq 0 .
$$


We next define two subspaces of $H^{1}$ where $\Phi$ is positive. Consider the following orthogonality relations:

$$
\begin{aligned}
& \int\left(\partial_{x_{1}} Q\right) h_{1}=\int\left(\partial_{x_{2}} Q\right) h_{1}=\int\left(\partial_{x_{3}} Q\right) h_{1}=\int Q h_{2}=0, \\
& \int \Delta Q h_{1}=0 \\
& \int \mathcal{Y}_{1} h_{2}=\int \mathcal{Y}_{2} h_{1}=0 .
\end{aligned}
$$

Let $G_{\perp}$ be the set of $h \in H^{1}$ satisfying the orthogonality relations (2.16) and (2.17) and $G_{\perp}^{\prime}$ the set of $h \in H^{1}$ satisfying (2.16) and (2.17'). Then

Proposition 2.7 (Coercivity of $\Phi)$. There exists a constant $c>0$ such that

$$
\forall h \in G_{\perp} \cup G_{\perp}^{\prime}, \quad \Phi(h) \geq c\|h\|_{H^{1}}^{2} .
$$

Unlike the energy-critical case, where the stationary solution $W$ is explicit, we cannot rely on explicit computations to prove Proposition 2.7. The main tool of the proof, which is postponed to Appendix A, is the GagliardoNirenberg inequality (2.2).

Observe that as a consequence of Proposition 2.7,

$$
\int \Delta Q \mathcal{Y}_{1} \neq 0
$$

Indeed, assume $\int \Delta Q \mathcal{Y}_{1}=0$. Then by (2.1) and direct computations, $\int L_{+} Q \mathcal{Y}_{1}=0$. By Remark 2.4 we obtain $\int Q \mathcal{Y}_{2}=0$ which shows that $Q$ is in $G_{\perp}^{\prime}$, contradicting $(2.15)$.

Remark 2.8. In [33] Weinstein gives a sharp description of the semi-group $e^{-t \mathcal{L}}$ for the mass-subcritical and mass-critical focusing NLS equations. In both cases, one may decompose $H^{1}$ as a direct sum $S \oplus M$, where $S$ and $M$ are stable by the flow of $e^{-t \mathcal{L}}, S$ is finite dimensional and contains the eigenfunctions of $\mathcal{L}$, and $\Phi$ is equivalent to the $H^{1}$ norm on $M$, which implies that $e^{-t \mathcal{L}} h_{0}$ is bounded in $H^{1}$ if $h_{0} \in M$. It is not clear whether such a convenient decomposition exists for the mass-supercritical NLS equation. Note that the vector space $G_{\perp}^{\prime}$, which will play the roles of $M$ in the sequel, is not invariant by the flow of the semi-group $e^{-t \mathcal{L}}$. However, Proposition 2.7 is sufficient for our needs, namely the description of the dynamics of exponentially decaying solutions of the linearized equation (see Subsection 7.1). 


\section{Existence of special solutions}

The aim of this section is to construct the solutions $Q^{+}$and $Q^{-}$of Theorem 2 . Namely, we will show:

Proposition 3.1. Let $A \in \mathbb{R}$. If $t_{0}=t_{0}(A)>0$ is large enough, then there exists a radial solution

$$
U^{A} \in C^{\infty}\left(\left[t_{0},+\infty\right), H^{\infty}\right)
$$

of (1.1) such that

(3.1) $\forall b \in \mathbb{R}, \exists C>0: \forall t \geq t_{0}$ we have

$$
\left\|U^{A}(t)-e^{i t} Q-A e^{\left(i-e_{0}\right) t} \mathcal{Y}_{+}\right\|_{H^{b}} \leq C e^{-2 e_{0} t} .
$$

Remark 3.2. Note that by (3.1), the conservation of mass and energy, we have

$$
M\left[U^{A}\right]=M[Q] \text {, and } E\left[U^{A}\right]=E[Q] .
$$

Furthermore, again by (3.1),

$$
\left\|\nabla U^{A}(t)\right\|_{2}^{2}=\|\nabla Q\|_{2}^{2}+2 A e^{-e_{0} t} \int \nabla Q \cdot \nabla \mathcal{Y}_{1}+O\left(e^{-2 e_{0} t}\right), \quad t \rightarrow+\infty .
$$

By (2.19), replacing $\mathcal{Y}_{+}$by $-\mathcal{Y}_{+}$if necessary, we may assume

$$
\int \nabla Q \nabla \mathcal{Y}_{1}>0
$$

which shows that $\left\|\nabla U^{A}(t)\right\|_{2}^{2}-\|\nabla Q\|_{2}^{2}$ has the sign of $A$ for large positive time. Thus, by Lemma 2.2, $\left\|\nabla U^{A}\left(t_{0}\right)\right\|_{2}^{2}-\|\nabla Q\|_{2}^{2}$ has the sign of $A$. Letting

$$
Q^{+}(x, t)=e^{-i t_{0}} U^{+1}\left(x, t+t_{0}\right), \quad Q^{-}(x, t)=e^{-i t_{0}} U^{-1}\left(x, t+t_{0}\right),
$$

we get two solutions satisfying

$$
\begin{aligned}
E[Q] & =E\left[Q^{ \pm}\right], \quad M[Q]=M\left[Q^{ \pm}\right], \\
\left\|\nabla Q^{+}(0)\right\|_{2}^{2} & >\|\nabla Q\|_{2}^{2}, \quad\left\|\nabla Q^{-}(0)\right\|_{2}^{2}<\|\nabla Q\|_{2}^{2}
\end{aligned}
$$

and

$$
\left\|Q^{ \pm}-e^{i t} Q\right\|_{H^{1}} \leq C e^{-e_{0} t}, \quad t \geq 0 .
$$

To conclude the proof of Theorem 2, it remains to specify the behavior of $Q^{+}$ and $Q^{-}$for negative $t$, which we will do in Remark 5.2 and $\S 6.4$. 
Remark 3.3. We will see in $\S 7.3$ that all solutions $U^{A}, A>0$ (respectively $A<0$ ) are equal to $Q^{+}$(respectively $Q^{-}$) up to a translation in time and a multiplication by a complex number of modulus 1 .

The proof of Proposition 3.1 is similar to the one of Proposition 6.1 in [8]. We start with the construction of a family of approximate solutions to (1.1) that satisfy (3.1), and then prove the existence of $U^{A}$ by a fixed point argument around an approximate solution.

\subsection{A family of approximate solutions}

Proposition 3.4. Let $A \in \mathbb{R}$. There exists a sequence $\left(\mathcal{Z}_{j}^{A}\right)_{j \geq 1}$ of functions in $\mathcal{S}$ such that $\mathcal{Z}_{1}^{A}=A \mathcal{Y}_{+}$and, if $k \geq 1$ and $\mathcal{V}_{k}^{A}:=\sum_{j=1}^{k} e^{-j e_{0} t} \mathcal{Z}_{j}^{A}$, then as $t \rightarrow+\infty$ we have

$$
\partial_{t} \mathcal{V}_{k}^{A}+\mathcal{L} \mathcal{V}_{k}^{A}=R\left(\mathcal{V}_{k}^{A}\right)+O\left(e^{-(k+1) e_{0} t}\right) \text { in } \mathcal{S},
$$

where the linear operator $\mathcal{L}$ and the nonlinear term $R$ are defined in (2.7).

Remark 3.5. Let $U_{k}^{A}:=e^{i t}\left(Q+\mathcal{V}_{k}^{A}\right)$. Then $U_{k}^{A}$ is an approximate solution of (1.1) for large $t$ and satisfies (3.1). Indeed, as $t \rightarrow+\infty$, we have

$$
i \partial_{t} U_{k}^{A}+\Delta U_{k}^{A}+\left|U_{k}^{A}\right|^{2} U_{k}^{A}=O\left(e^{-(k+1) e_{0} t}\right) \text { in } \mathcal{S} .
$$

Proof of Proposition 3.4. We prove this proposition by induction. For brevity, we omit the superscript $A$.

Define $\mathcal{Z}_{1}:=A \mathcal{Y}_{+}$and $\mathcal{V}_{1}:=e^{-e_{0} t} \mathcal{Z}_{1}$. Then

$$
\partial_{t} \mathcal{V}_{1}+\mathcal{L} \mathcal{V}_{1}-R\left(\mathcal{V}_{1}\right)=-R\left(\mathcal{V}_{1}\right)=-R\left(e^{-e_{0} t} \mathcal{Z}_{1}\right)
$$

which yields (3.2) for $k=1$.

Let $k \geq 1$ and assume that $\mathcal{Z}_{1}, \ldots, \mathcal{Z}_{k}$ are known with the corresponding $\mathcal{V}_{k}$ satisfying (3.2). Expand the expression of $R\left(\mathcal{V}_{k}\right)$ by using (2.9), and observe that $R\left(\mathcal{V}_{k}\right)$ is of the form $\sum_{j=2}^{3 k} e^{-j e_{0} t} f_{j k}$ with $f_{j k}$ 's being in $\mathcal{S}$. Thus, by (3.2), there exists $\mathcal{U}_{k+1} \in \mathcal{S}$ such that, as $t \rightarrow+\infty$, we have

$$
\partial_{t} \mathcal{V}_{k}+\mathcal{L} \mathcal{V}_{k}=R\left(\mathcal{V}_{k}\right)+e^{-(k+1) e_{0} t} \mathcal{U}_{k+1}+O\left(e^{-(k+2) e_{0} t}\right) \text { in } \mathcal{S}
$$

By Proposition 2.3, $(k+1) e_{0}$ is not in the spectrum of $\mathcal{L}$. Define

$$
\mathcal{Z}_{k+1}:=-\left(\mathcal{L}-(k+1) e_{0}\right)^{-1} \mathcal{U}_{k+1} .
$$

It is classical that $\mathcal{Z}_{k+1} \in \mathcal{S}$ (e.g., see [8, Appendix 7.2.2] for an elementary proof in a similar setting). Then we have

$$
\begin{aligned}
\partial_{t}\left(\mathcal{V}_{k}+e^{-(k+1) e_{0} t} \mathcal{Z}_{k+1}\right)+\mathcal{L}\left(\mathcal{V}_{k}+e^{-(k+1) e_{0} t} \mathcal{Z}_{k+1}\right) & \\
& =R\left(\mathcal{V}_{k}\right)+O\left(e^{-(k+2) e_{0} t}\right) \text { in } \mathcal{S} .
\end{aligned}
$$


Denote $\mathcal{V}_{k+1}:=\mathcal{V}_{k}+e^{-(k+1) e_{0} t} \mathcal{Z}_{k+1}$. By (3.3), $\mathcal{V}_{k+1}$ satisfies, as $t \rightarrow+\infty$,

$$
\partial_{t} \mathcal{V}_{k+1}+\mathcal{L} \mathcal{V}_{k+1}-R\left(\mathcal{V}_{k+1}\right)=R\left(\mathcal{V}_{k}\right)-R\left(\mathcal{V}_{k+1}\right)+O\left(e^{-(k+2) e_{0} t}\right) \text { in } \mathcal{S}
$$

Since we have, as $t \rightarrow+\infty, \mathcal{V}_{j}=O\left(e^{-e_{0} t}\right)$ in $\mathcal{S}$ for $j=k, k+1$ and $\mathcal{V}_{k}-\mathcal{V}_{k+1}=O\left(e^{-(k+1) e_{0} t}\right)$ in $\mathcal{S}$, we obtain (using the explicit expression of $R$ ),

$$
R\left(\mathcal{V}_{k}\right)-R\left(\mathcal{V}_{k+1}\right)=O\left(e^{-(k+2) e_{0} t}\right) \text { in } \mathcal{S}
$$

as $t \rightarrow+\infty$ which shows, in view of (3.4), the desired estimate (3.2) for $k+1$. This completes the proof.

\subsection{Construction of special solutions}

Next we prove Proposition 3.1. We will construct a solution $U^{A}$ such that there exists $t_{0} \in \mathbb{R}$

$$
\begin{aligned}
\forall b \in \mathbb{R}, \exists C>0: \forall t \geq & t_{0} \text { and } \forall k \in \mathbb{N} \\
& \left\|U^{A}(t)-e^{i t}\left(Q+\mathcal{V}_{k}^{A}(t)\right)\right\|_{H^{b}} \leq C e^{-(k+1) e_{0} t}
\end{aligned}
$$

Let $b>3 / 2$ and write

$$
U^{A}=e^{i t}\left(Q+h^{A}\right)
$$

First, by a fixed point argument we construct a solution of (2.7) $h^{A} \in$ $C^{0}\left(\left[t_{k},+\infty\right), H^{b}\right)$ for $k$ and $t_{k}$ large and such that

$$
\forall t \geq t_{k}, \quad\left\|h^{A}(t)-\mathcal{V}_{k}^{A}(t)\right\|_{H^{b}} \leq e^{-\left(k+\frac{1}{2}\right) e_{0} t} .
$$

Next, we show by uniqueness arguments that $h^{A}$ does not depend on $b$ and $k$. Estimate (3.5) will follow from (3.6). For brevity we again omit the superscript $A$.

Step 1. Reduction to a fixed point problem. The equation (2.7) may be written as a Schrödinger equation

$$
i \partial_{t} h+\Delta h-h=-S(h), \quad S(h):=2 Q^{2} h+Q^{2} \bar{h}+2 Q|h|^{2}+Q h^{2}+|h|^{2} h .
$$

For $k \in \mathbb{N}$ define

$$
\varepsilon_{k}(t)=i \partial_{t} \mathcal{V}_{k}+\Delta \mathcal{V}_{k}-\mathcal{V}_{k}+S\left(\mathcal{V}_{k}\right)
$$

By Proposition 3.4, as $t \rightarrow+\infty$,

$$
\varepsilon_{k}(t)=O\left(e^{-(k+1) e_{0} t}\right) \text { in } \mathcal{S} .
$$


Let $v:=h-\mathcal{V}_{k}$ and subtract (3.8) from (3.7) to obtain

$$
i \partial_{t} v+\Delta v-v=-S\left(\mathcal{V}_{k}+v\right)+S\left(\mathcal{V}_{k}\right)-\varepsilon_{k} .
$$

The solution of (3.10) is given by the equation

$$
v(t)=\mathcal{M}(v)(t),
$$

where

$$
\mathcal{M}(v)(t):=-i \int_{t}^{+\infty} e^{i(t-s)(\Delta-1)}\left[S\left(\mathcal{V}_{k}(s)+v(s)\right)-S\left(\mathcal{V}_{k}(s)\right)+\varepsilon_{k}(s)\right] d s .
$$

Note that (3.6) is equivalent to $\|v(t)\|_{H^{b}} \leq e^{-\left(k+\frac{1}{2}\right) e_{0} t}$ for $t \geq t_{k}$. Thus, we must show that $\mathcal{M}$ is a contraction on $B$ defined by

$$
\begin{aligned}
B & =B\left(t_{k}, k, b\right):=\left\{v \in E,\|v\|_{E} \leq 1\right\}, \\
E & =E\left(t_{k}, k, b\right) \\
& :=\left\{v \in C^{0}\left(\left[t_{k},+\infty\right), H^{b}\right),\|v\|_{E}=\sup _{t \geq t_{k}} e^{\left(k+\frac{1}{2}\right) e_{0} t}\|v(t)\|_{H^{b}}<\infty\right\} .
\end{aligned}
$$

This is the object of the following step.

Step 2. Contraction argument. We show that $\mathcal{M}$ is a contraction on $B$ for $b>3 / 2$, and $k$ and $t_{k}$ sufficiently large ${ }^{4}$.

Throughout this proof, we denote by $C$ a constant depending only on $b$, and $C_{k}$ a constant depending on $b$ and $k$. Both constants may change from line to line. Note that $H^{b}$ is closed under multiplication and conjugation for $b>\frac{3}{2}$. In view of the identities

$$
\begin{gathered}
F^{2}-G^{2}=(F-G)(F+G), \quad|F|^{2}-|G|^{2}=\operatorname{Re}((\bar{F}-\bar{G})(F+G)), \\
|F|^{2} F-|G|^{2} G=F \operatorname{Re}((\bar{F}-\bar{G})(F+G))+|G|^{2}(F-G),
\end{gathered}
$$

we obtain that for $F, G \in H^{b}$ there exists a constant $C_{0}>0$ such that

$$
\|S(F)-S(G)\|_{H^{b}} \leq C_{0}\|F-G\|_{H^{b}}\left(1+\|F\|_{H^{b}}^{2}+\|G\|_{H^{b}}^{2}\right) .
$$

Let $v \in B$. Observe that for all $t \in \mathbb{R}, e^{i t(\Delta-1)}$ is an isometry of $H^{b}$. By the definition of $\mathcal{M}$, and applying the bound (3.9) on $\varepsilon_{k}$ and the estimate (3.12), we get

(3.13) $\forall t \geq t_{k}, \quad\|\mathcal{M}(v(t))\|_{H^{b}}$

$$
\leq C \int_{t}^{+\infty}\|v(s)\|_{H^{b}}\left(1+\left\|\mathcal{V}_{k}(s)\right\|_{H^{b}}^{2}+\|v(s)\|_{H^{b}}^{2}\right) d s+C_{k} \int_{t}^{+\infty} e^{-(k+1) e_{0} s} d s .
$$

\footnotetext{
${ }^{4}$ Note that the condition $b>3 / 2$ is not restrictive: if $(3.5)$ is shown for some $b_{0}$, it follows for all $b \leq b_{0}$.
} 
By the construction of $\mathcal{V}_{k},\left\|\mathcal{V}_{k}(s)\right\|_{H^{b}} \leq C_{k} e^{-e_{0} s}$. Furthermore, since $v \in B$, $\|v(s)\|_{H^{b}} \leq e^{-\left(k+\frac{1}{2}\right) e_{0} s}$. Hence,

$$
\begin{aligned}
\forall t \geq t_{k}, \quad \int_{t}^{+\infty}\|v(s)\|_{H^{b}} & \left(1+\left\|\mathcal{V}_{k}(s)\right\|_{H^{b}}^{2}+\|v(s)\|_{H^{b}}^{2}\right) d s \\
& \leq 2 \int_{t}^{+\infty}\left(e^{-\left(k+\frac{1}{2}\right) e_{0} s}+C_{k} e^{-\left(k+\frac{5}{2}\right) e_{0} s}\right) d s \\
& \leq\left(\frac{2}{\left(k+\frac{1}{2}\right) e_{0}}+C_{k} e^{-2 e_{0} t}\right) e^{-\left(k+\frac{1}{2}\right) e_{0} t} .
\end{aligned}
$$

Therefore, $\mathcal{M}(v) \in E$, and from (3.13) we obtain

$$
\|\mathcal{M}(v)\|_{E} \leq \frac{C}{k+\frac{1}{2}}+C_{k} e^{-\frac{1}{2} e_{0} t_{k}} .
$$

First, choose $k$ so that $\frac{C}{k+\frac{1}{2}} \leq \frac{1}{2}$; next, take $t_{k}$ such that $C_{k} e^{-\frac{1}{2} e_{0} t_{k}} \leq \frac{1}{2}$. Then $\mathcal{M}$ maps $B=B\left(t_{k}, k, b\right)$ into itself.

It remains to show that $\mathcal{M}$ is a contraction. Let $v, w \in B$. By the definition of $\mathcal{M}$ and (3.12), we have

$$
\begin{aligned}
\|\mathcal{M}(v)(t)-\mathcal{M}(w)(t)\|_{H^{b}} & \leq \int_{t}^{+\infty}\left\|S\left(\mathcal{V}_{k}(s)+v(s)\right)-S\left(\mathcal{V}_{k}(s)+w(t)\right)\right\|_{H^{b}} d s \\
& \leq \int_{t}^{+\infty}\left(C+C_{k} e^{-2 e_{0} s}\right)\|v(s)-w(s)\|_{H^{b}} d s \\
& \leq\left(\frac{C}{k+\frac{1}{2}}+C_{k} e^{-2 e_{0} t_{k}}\right) e^{-\left(k+\frac{1}{2}\right) t}\|v-w\|_{E} .
\end{aligned}
$$

Choosing if necessary a larger $k$, then a larger $t_{k}$, we may assume that $\frac{C}{k+\frac{1}{2}}<\frac{1}{2}$ and $C_{k} e^{-2 e_{0} t_{k}} \leq \frac{1}{2}$, showing that $\mathcal{M}$ is a contraction on $B$. Hence, Step 2 is complete.

Step 3. End of the proof. By the preceding step with $b=2$, there exists $k_{0}$ and $t_{0}$ such that there exists a unique solution $U^{A}$ of (1.1) satisfying $U^{A} \in C^{0}\left(\left[t_{0},+\infty\right), H^{2}\right)$ and for all $t \geq t_{0}$

$$
\left\|U^{A}(t)-e^{i t} Q-e^{i t} \mathcal{V}_{k_{0}}^{A}(t)\right\|_{H^{2}} \leq e^{-\left(k_{0}+\frac{1}{2}\right) e_{0} t}
$$

Note that the fixed point argument still holds taking a larger $t_{0}$, so that the uniqueness remains valid, for any $t_{0}^{\prime} \geq t_{0}$, in the class of solutions of (1.1) in $C^{0}\left(\left[t_{0}^{\prime},+\infty\right), H^{2}\right)$ satisfying (3.14) for all $t \geq t_{0}^{\prime}$.

Next, we show that $U^{A} \in C^{\infty}\left(\left[t_{0},+\infty\right), H^{b}\right)$ and that (3.5) holds for any $b \in \mathbb{R}$. 
Since $U^{A}$ is a solution of (1.1), it is sufficient to show that $U^{A}$ is in $C^{0}\left(\left[t_{0},+\infty\right), H^{b}\right)$ for any $b$; the smoothness in time will follow from (1.1) and Sobolev embeddings. Let $b \geq 2$. By Step 2, if $k_{1}$ is large enough, there exists $t_{1}$ and $\widetilde{U}^{A} \in C^{0}\left(\left[t_{1},+\infty\right), H^{b}\right)$ such that

$$
\forall t \geq t_{1}, \quad\left\|\widetilde{U}^{A}-e^{i t} Q-e^{i t} \mathcal{V}_{k_{1}}^{A}(t)\right\|_{H^{b}} \leq e^{-\left(k_{1}+\frac{1}{2}\right) e_{0} t}
$$

Of course, we may choose $k_{1} \geq k_{0}+1$. Using that by the construction of $\mathcal{V}_{k}^{A}$,

$$
\left\|\mathcal{V}_{k_{1}}^{A}-\mathcal{V}_{k_{0}}^{A}\right\|_{H^{b}} \leq C e^{-\left(k_{0}+1\right) e_{0} t}
$$

we get

$$
\begin{aligned}
& \forall t \geq t_{1},\left\|\widetilde{U}^{A}-e^{i t} Q-e^{i t} \mathcal{V}_{k_{0}}^{A}(t)\right\|_{H^{b}} \leq \\
& \quad \leq e^{-\left(k_{1}+\frac{1}{2}\right) e_{0} t}+C e^{-\left(k_{0}+1\right) e_{0} t} \leq C e^{-\left(k_{0}+1\right) e_{0} t}
\end{aligned}
$$

In particular, $\widetilde{U}^{A}$ satisfies (3.14) for large $t$. By the uniqueness in the fixed point argument, $U^{A}=\widetilde{U}^{A}$, which shows that $U^{A} \in C^{0}\left(\left[t_{1},+\infty\right), H^{b}\right), b \geq 2$. By the persistence of regularity of equation (1.1), $U^{A} \in C^{0}\left(\left[t_{0},+\infty\right), H^{b}\right)$, $b \geq 2$ (and thus for any $b \in \mathbb{R}$ ). Finally, we note that (3.15) implies (3.5) with $k_{0}$ replaced by $k$, which concludes the proof of Proposition 3.1.

Remark 3.6. The estimate (3.5) yields an asymptotic expansion of $U^{A}$ in terms of $e^{-e_{0} t}$.

\section{Modulation}

For $u \in H^{1}$ define

$$
\delta(u)=\left.\left|\int\right| \nabla Q\right|^{2}-\int|\nabla u|^{2} \mid .
$$

By Proposition 2.1, if

$$
M[u]=M[Q], \quad E[u]=E[Q],
$$

and $\delta(u)$ is small enough, then there exists $\tilde{\theta}$ and $\tilde{X}$ such that $e^{-i \tilde{\theta}} u(\cdot+\tilde{X})=$ $Q+\tilde{u}$ with $\|\tilde{u}\|_{H^{1}} \leq \tilde{\varepsilon}(\delta(u))$, where $\tilde{\varepsilon}(\delta) \rightarrow 0$ as $\delta \rightarrow 0$. Recall that any solution such that (1.3) holds may be rescaled to a solution satisfying (4.1). The goal of this section is to choose parameters $\tilde{\theta}$ and $\tilde{X}$, when $u$ is a solution of (1.1), in order to obtain linear estimates of these parameters and their derivatives in terms of $\delta(u)$. 
Lemma 4.1. There exist $\delta_{0}>0$ and a positive function $\varepsilon(\delta)$ defined for $0<\delta \leq \delta_{0}$, which tends to 0 when $\delta$ tends to 0 , such that for all $u$ in $H^{1}\left(\mathbb{R}^{3}\right)$ satisfying $(4.1)$ and $\delta(u)<\delta_{0}$, there exists a couple $(\sigma, X)$ in $\mathbb{R} \times \mathbb{R}^{3}$ such that $v=e^{-i \sigma} u(\cdot+X)$ satisfies

$$
\begin{gathered}
\|v-Q\|_{H^{1}} \leq \varepsilon(\delta), \\
\operatorname{Im} \int Q v=0, \quad \operatorname{Re} \int \partial_{x_{k}} Q v=0, k=1,2,3 .
\end{gathered}
$$

The parameters $\sigma$ and $X$ are unique in $\mathbb{R} / 2 \pi \mathbb{Z} \times \mathbb{R}^{3}$, and the mapping $u \mapsto$ $(\sigma, X)$ is $C^{1}$.

Proof. Let us first show the lemma when $u$ is close to $Q$ in $H^{1}$. Consider the following functionals on $\mathbb{R} \times \mathbb{R}^{3} \times H^{1}$ :

$$
\begin{aligned}
& J_{0}:(\sigma, X, u) \mapsto \operatorname{Im} \int e^{-i \sigma} u(x+X) Q \\
& J_{k}:(\sigma, X, u) \mapsto \operatorname{Re} \int e^{-i \sigma} u(x+X) \partial_{x_{k}} Q, k=1,2,3 .
\end{aligned}
$$

Denote $J=\left(J_{0}, J_{1}, J_{2}, J_{3}\right)$. The orthogonality conditions (4.3) are equivalent to the condition $J(\sigma, X, u)=0$.

Note that $J(0,0, Q)=0$. Furthermore, by direct computation and using that since $Q$ is radial, $\int \partial_{x_{k}} Q \partial_{x_{j}} Q=0$ if $j \neq k$, one can check that $\left(\frac{\partial J}{\partial \sigma}, \frac{\partial J}{\partial X_{1}}, \frac{\partial J}{\partial X_{2}}, \frac{\partial J}{\partial X_{3}}\right)$ is invertible at $(0,0, Q)$. By the Implicit Function Theorem there exists $\varepsilon_{0}, \eta_{0}>0$ such that for $u \in H^{1}$

$$
\|u-Q\|_{H^{1}}<\varepsilon_{0} \Longrightarrow \exists !(\sigma, X), \quad|\sigma|+|X| \leq \eta_{0} \text { and } J(\sigma, X, u)=0 .
$$

If $u$ is as in the Lemma, we reduce the proof to the previous case by choosing $\tilde{\theta}$ and $\tilde{X}$ as in the introduction of this section, so that $e^{-i \tilde{\theta}} u(\cdot+\tilde{X})$ is close to $Q$ in $H^{1}$. The assertions on the uniqueness of $(\sigma, X)$ and the regularity of the mapping $u \mapsto(\sigma, X)$ follows from the Implicit Functions Theorem.

Let $u$ be a solution of (1.1) satisfying (4.1). In the sequel we will write

$$
\delta(t):=\delta(u(t))
$$

Let $D_{\delta_{0}}$ be the open set of all times $t$ in the domain of existence of $u$ such that $\delta(t)<\delta_{0}$. On $D_{\delta_{0}}$, by Lemma 4.1 we can define parameters $\sigma(t), X(t)$, which are $C^{1}$ functions of $t$. In the forthcoming sections, we show under the additional hypothesis that $u$ is close to $e^{i t} Q$, up to constant modulation 
parameters, and thus, we rather work with the parameters $X(t)$ and $\theta(t)=$ $\sigma(t)-t$. Write

$$
\begin{aligned}
& e^{-i \theta(t)-i t} u(t, x+X(t))=(1+\alpha(t)) Q(x)+h(t, x), \\
& \alpha(t)=\operatorname{Re} \frac{e^{-i \theta-i t} \int \nabla u(t, x+X(t)) \cdot \nabla Q(x) d x}{\int|\nabla Q|^{2}}-1 .
\end{aligned}
$$

Observe that $\alpha$ is chosen such that $h$ satisfies the orthogonality condition (2.17). By Lemma 4.1, $h$ also satisfies the orthogonality conditions (2.16).

We next obtain a first estimate on the parameters.

Lemma 4.2. Let $u$ be a solution of (1.1) satisfying (4.1). Then, taking a smaller $\delta_{0}$ if necessary, the following estimates hold for $t \in D_{\delta_{0}}$ :

$$
|\alpha(t)| \approx\left|\int Q h_{1}(t)\right| \approx\|h(t)\|_{H^{1}} \approx \delta(t) .
$$

Proof. Let $\tilde{\delta}(t):=|\alpha(t)|+\delta(t)+\|h(t)\|_{H^{1}}$, which is small when $\delta(t)$ is small (see (4.2)). The equality $M[Q+\alpha Q+h]=M[u]=M[Q]$ implies $\int|\alpha Q+h|^{2}+2 \alpha \int Q^{2}+2 \int Q h_{1}=0$, and hence,

$$
|\alpha|=\frac{1}{M[Q]}\left|\int Q h_{1}\right|+O\left(\tilde{\delta}^{2}\right)
$$

Furthermore, by definition of $\delta$ and the orthogonality condition (2.17) on $h$, we obtain

$\delta(t)=\left.\left|\int\right| \nabla(Q+\alpha Q+h)\right|^{2}-\int|\nabla Q|^{2}|=|\left(2 \alpha+\alpha^{2}\right) \int|\nabla Q|^{2}+\int|\nabla h|^{2} \mid$,

which yields

$$
|\alpha|=\frac{1}{2\|\nabla Q\|^{2}} \delta+O\left(\tilde{\delta}^{2}\right)
$$

Note that the orthogonality condition $\int \nabla Q \cdot \nabla h_{1}=0$ implies with equation (2.1) that $\int Q^{3} h_{1}=\int Q h_{1}$. Thus, $B(Q, h)=-\int Q h_{1}$, where $B$ is as in (2.13). By (2.12),

$$
\begin{gathered}
\left|\alpha^{2} \Phi(Q)+\Phi(h)-2 \alpha \int Q h_{1}\right|=|\Phi(\alpha Q+h)|=O\left(\alpha^{3}+\|h\|_{H^{1}}^{3}\right), \\
\Phi(h)=\alpha^{2}|\Phi(Q)|+2 \alpha \int Q h_{1}+O\left(\alpha^{3}+\|h\|_{H^{1}}^{3}\right) .
\end{gathered}
$$


By Proposition 2.7 and $(2.12), \Phi(h) \approx\|h\|_{H^{1}}^{2}$. Combining this and (4.8), we get

$$
\|h\|_{H^{1}}=O\left(|\alpha|+\left|\int Q h_{1}\right|+\tilde{\delta}^{3 / 2}\right) .
$$

Substituting (4.6) into (4.9) we get $\|h\|_{H^{1}}=O\left(|\alpha|+\tilde{\delta}^{3 / 2}\right)$ and thus, with (4.7), $\tilde{\delta}=O(|\alpha|)$, which shows that (4.6), (4.7) and (4.9) imply (4.5).

In the sequel we denote by ' the derivative with respect to $t$.

Lemma 4.3 (Bounds on the time-derivatives). Under the assumption of Lemma 4.2, taking a smaller $\delta_{0}$ if necessary, we have for $t \in D_{\delta_{0}}$

$$
\left|\alpha^{\prime}\right|+\left|X^{\prime}\right|+\left|\theta^{\prime}\right|=O(\delta) .
$$

Proof. Let $\delta^{*}(t)=\delta(t)+\left|\alpha^{\prime}(t)\right|+\left|X^{\prime}(t)\right|+\left|\theta^{\prime}(t)\right|$. The equation (1.1) and Lemma 4.2 yield for $t \in D_{\delta_{0}}$

$$
i \partial_{t} h+\Delta h+i \alpha^{\prime} Q-\theta^{\prime} Q-i X^{\prime} \cdot \nabla Q=O\left(\delta+\delta \delta^{*}\right) \text { in } L^{2} .
$$

Note that by orthogonality relations (2.16) and (2.17) on $h$, we have

$$
\begin{aligned}
\forall t \in D_{\delta_{0}}, \quad \operatorname{Re} \int \partial_{x_{j}} Q \partial_{t} h=0, j=1,2,3, \\
\text { and } \operatorname{Im} \int Q \partial_{t} h=\operatorname{Re} \int \Delta Q \partial_{t} h=0 .
\end{aligned}
$$

Multiplying (4.11) by $Q$, integrating the real part on $\mathbb{R}^{3}$, we get by integration by parts (and using that by $(2.17) \operatorname{Re} \int h \Delta Q=0$ ),

$$
\left|\theta^{\prime}\right|=O\left(\delta+\delta \delta^{*}\right)
$$

Similarly, multiplying (4.11) by $\partial_{x_{j}} Q, j \in\{1,2,3\}$, and integrating the imaginary part on $\mathbb{R}^{3}$, we obtain (using that by Lemma $4.2, \int \Delta h \partial_{x_{j}} Q=O(\delta)$ ),

$$
\left|X_{j}^{\prime}\right|=O\left(\delta+\delta \delta^{*}\right)
$$

where $X=\left(X_{1}, X_{2}, X_{3}\right)$. Multiplying (4.11) by $\Delta Q$ and taking the imaginary part we get (noting that $\int \partial_{x_{j}} Q \Delta Q=0$ and that by Lemma 4.2, $\left.\int \Delta h \partial_{x_{j}} Q=O(\delta)\right)$,

$$
\left|\alpha^{\prime}\right|=O\left(\delta+\delta \delta^{*}\right) .
$$

Summing up (4.13), (4.14) and (4.15), we obtain

$$
\delta^{*}=O\left(\delta+\delta \delta^{*}\right),
$$

which yields the result if $\delta_{0}$ is chosen small enough.

We conclude this section by showing the following Lemma, needed in the next two sections. 
Lemma 4.4. Let $u$ be a solution of (1.1) satisfying (4.1). Assume that $u$ is defined on $[0,+\infty)$ and that there exist $c, C>0$ such that

$$
\forall t \geq 0, \quad \int_{t}^{+\infty} \delta(s) d s \leq C e^{-c t} .
$$

Then there exist $\theta_{0} \in \mathbb{R}, x_{0} \in \mathbb{R}^{3}, c, C>0$ such that

$$
\left\|u-e^{i t+i \theta_{0}} Q\left(\cdot-x_{0}\right)\right\|_{H^{1}} \leq C e^{-c t} .
$$

Proof. First observe that (4.16) implies that there exists $\left\{t_{n}\right\}_{n \in \mathbb{N}}$ with $t_{n} \rightarrow+\infty$ such that

$$
\lim _{n \rightarrow+\infty} \delta\left(t_{n}\right)=0
$$

If not, there exists $\varepsilon>0$ such that $\delta(t) \geq \varepsilon$ for a.e. $t>0$, which would contradict (4.16).

Fix such $\left\{t_{n}\right\}_{n \in \mathbb{N}}$. Next we show that $\delta(t)$ tends to 0 as $t$ tends to $+\infty$. If not, there exists a sequence $\left\{t_{n}^{\prime}\right\}_{n \in \mathbb{N}}$ which tends to $+\infty$ such that $\delta\left(t_{n}^{\prime}\right) \geq$ $\varepsilon_{1}>0$ for some $\varepsilon_{1}>0$. Now we can choose $\left\{t_{n}^{\prime}\right\}$, extracting subsequences from $\left\{t_{n}\right\}$ and/or $\left\{t_{n}^{\prime}\right\}$ if necessary, with the following properties:

$$
\begin{aligned}
& \forall n, \quad t_{n}<t_{n}^{\prime} \\
& \delta\left(t_{n}^{\prime}\right)=\varepsilon_{1},
\end{aligned}
$$

and

$$
\forall t \in\left[t_{n}, t_{n}^{\prime}\right) \quad \delta(t)<\varepsilon_{1} .
$$

On $\left[t_{n}, t_{n}^{\prime}\right]$ the parameter $\alpha(t)$ is well defined. By Lemma 4.3, $\left|\alpha^{\prime}(t)\right|=$ $O(\delta(t))$, so that by $(4.16), \int_{t_{n}}^{t_{n}^{\prime}}\left|\alpha^{\prime}(t)\right| d t \leq C e^{-c t_{n}}$. Hence,

$$
\lim _{n \rightarrow+\infty}\left|\alpha\left(t_{n}\right)-\alpha\left(t_{n}^{\prime}\right)\right|=0 .
$$

By Lemma 4.2, we have $|\alpha(t)| \approx \delta(t)$. As a consequence, (4.17) implies that $\left|\alpha\left(t_{n}\right)\right|$ tends to 0 , however, (4.19) implies that $\left|\alpha\left(t_{n}^{\prime}\right)\right|$ is bounded from below as $t$ tends to $+\infty$. This contradicts (4.21) and shows as announced

$$
\lim _{t \rightarrow+\infty} \delta(t)=0
$$

To conclude the proof of Lemma 4.4, in view of the decomposition (4.4) of $u$, it is sufficient to show that there exists $\theta_{\infty}, X_{\infty}$ such that

$$
\forall t \geq 0, \quad \delta(t)+|\alpha(t)|+\|h(t)\|_{H^{1}}+\left|X(t)-X_{\infty}\right|+\left|\theta(t)-\theta_{\infty}\right| \leq C e^{-c t}
$$


Note that by (4.22) and the estimate $|\alpha(t)| \approx \delta(t), \alpha(t)$ tends to 0 as $t$ tends to $+\infty$, and thus, $\alpha(t)=-\int_{t}^{\infty} \alpha^{\prime}(s) d s$. By (4.16) and the estimate $\left|\alpha^{\prime}(t)\right|=O(\delta(t))$, we get the bound on $\alpha(t)$ in (4.23). Since by Lemma 4.2, $|\alpha(t)| \approx\|h(t)\|_{H^{1}} \approx \delta(t)$, we deduce the bound on $\delta$ and $h$. From Lemma 4.3, we get $\left|X^{\prime}(t)\right|+\left|\theta^{\prime}(t)\right| \leq C e^{-c t}$. Thus, there exist $X_{\infty}, \theta_{\infty}$ such that $\mid X(t)-$ $X_{\infty}|+| \theta(t)-\theta_{\infty} \mid \leq C e^{-c t}$, concluding the proof of (4.23).

\section{Convergence to $Q$ in the case $\left\|\nabla u_{0}\right\|_{2}\left\|u_{0}\right\|_{2}>\|\nabla Q\|_{2}\|Q\|_{2}$}

In this section, we show the following proposition, which is the first step in the proof of case (c) of Theorem 3 .

Proposition 5.1. Consider a solution $u$ of (1.1) such that

$$
\begin{gathered}
M[u]=M[Q], \quad E[u]=E[Q], \\
\left\|\nabla u_{0}\right\|_{2}>\|\nabla Q\|_{2},
\end{gathered}
$$

which is globally defined for positive times. Assume furthermore that $u_{0}$ is either of finite variance, i.e.,

$$
\int|x|^{2}\left|u_{0}(x)\right|^{2} d x<\infty
$$

or radial. Then there exists $\theta_{0} \in \mathbb{R}, x_{0} \in \mathbb{R}^{3}, c, C>0$ such that

$$
\left\|u-e^{i t+i \theta_{0}} Q\left(\cdot-x_{0}\right)\right\|_{H^{1}} \leq C e^{-c t} .
$$

Moreover, the negative time of existence of $u$ is finite.

Remark 5.2. The last statement of Proposition 5.1 shows that the radial solution $Q^{+}$constructed in Remark 3.2 has finite negative time of existence.

\subsection{Finite variance solutions}

In the finite variance case, Proposition 5.1 relies on the following lemma.

Lemma 5.3. Let $u$ be a solution of (1.1) satisfying (5.1), (5.2), (5.3) and

$$
T_{+}\left(u_{0}\right)=+\infty \text {. }
$$

Then for all $t$ in the interval of existence of $u$,

$$
\operatorname{Im} \int x \cdot \nabla u(x, t) \bar{u}(x, t) d x>0,
$$

and there exist $c, C>0$ such that

$$
\forall t \geq 0, \quad \int_{t}^{\infty} \delta(s) d s \leq C e^{-c t} .
$$


Let us first assume Lemma 5.3 and prove Proposition 5.1 in the finite variance case.

By (5.5), $\operatorname{Im} \int x \cdot \nabla u(x, t) \bar{u}(x, t) d x>0$ for all $t$ in the interval of existence of $u$. Now assume that $u$ is also globally defined for negative times, and consider the function $v(x, t)=\bar{u}(x,-t)$. Then $v$ is a solution of (1.1) satisfying the assumptions of Lemma 5.3. Thus, by (5.5), for all $t$ in the domain of existence of $u$,

$$
0<\operatorname{Im} \int x \cdot \nabla v(x,-t) \bar{v}(x,-t)=-\operatorname{Im} \int x \cdot \nabla u(x, t) \bar{u}(x, t) d x,
$$

which contradicts (5.5). Hence, the negative time of existence of $u$ is finite. The other assertion of Proposition 5.1 follows from (5.6) and Lemma 4.4.

To finish Subsection 5.1, we must prove Lemma 5.3.

Proof of Lemma 5.3. Let $y(t):=\int|x|^{2}|u(x, t)|^{2} d x$. Then by equation (1.1) and direct computations, we have, for all $t$ in the interval of existence of the solution $u$

$$
y^{\prime}(t)=4 \operatorname{Im} \int x \cdot \nabla u \bar{u} d x .
$$

Furthermore, $y^{\prime \prime}(t)=8 \int|\nabla u|^{2}-6 \int|u|^{4}$. By (2.4), $\|Q\|_{4}^{4}=\frac{4}{3}\|\nabla Q\|_{2}^{2}$. Using that $E[u]=E[Q]$, we get $8 \int|\nabla u|^{2}-6 \int|u|^{4}=4\left(\|\nabla Q\|_{2}^{2}-\|\nabla u\|_{2}^{2}\right)$, and thus,

$$
y^{\prime \prime}(t)=-4 \delta(t)<0
$$

Step 1. We first show (5.5), which is equivalent to

$$
y^{\prime}(t)>0 .
$$

If not, there exists $t_{1}$ such that $y^{\prime}\left(t_{1}\right) \leq 0$. By (5.8), if $t_{0}>t_{1}$ we obtain

$$
\forall t \geq t_{0}, \quad y^{\prime}(t) \leq y^{\prime}\left(t_{0}\right)<0 .
$$

As $T_{+}\left(u_{0}\right)=+\infty$, this shows that $y(t)<0$ for large $t$, yielding a contradiction.

Step 2. End of the proof. We first note that

$$
\left(y^{\prime}(t)\right)^{2} \leq C y(t)\left(y^{\prime \prime}(t)\right)^{2} .
$$

Indeed this is an immediate consequence of (5.7), (5.8) and the following claim, in the spirit of [2, Lemma 2.1], proven in Appendix B. 
Claim 5.4. Let $\varphi \in C^{1}\left(\mathbb{R}^{3}\right)$ and $f \in H^{1}\left(\mathbb{R}^{3}\right)$. Assume that $\int|f|^{2}|\nabla \varphi|^{2}$ is finite and

$$
\|f\|_{2}=\|Q\|_{2}, \quad E[f]=E[Q] .
$$

Then

$$
\left|\operatorname{Im} \int(\nabla \varphi \cdot \nabla f) \bar{f}\right|^{2} \leq C \delta^{2}(f) \int|\nabla \varphi|^{2}|f|^{2} .
$$

Taking $\varphi(x)=|x|^{2}$ in the above Claim, we obtain (5.11).

Now, for all $t$ in the interval of existence of $u$ we get $y^{\prime}(t)>0$ and $y^{\prime \prime}(t)<0$ (see (5.9) and (5.8)). Thus,

$$
\frac{y^{\prime}(t)}{\sqrt{y(t)}} \leq-C y^{\prime \prime}(t) .
$$

Integrating between 0 and $t \geq 0$, we get

$$
\sqrt{y(t)}-\sqrt{y(0)} \leq-C\left(y^{\prime}(t)-y^{\prime}(0)\right) \leq C y^{\prime}(0) .
$$

This shows that $y(t)$ is bounded for $t \geq 0$. From (5.14) we deduce

$$
y^{\prime}(t) \leq-C y^{\prime \prime}(t)
$$

which shows

$$
y^{\prime}(t) \leq C e^{-c t}
$$

Now

$$
y^{\prime}(t)=-\int_{t}^{\infty} y^{\prime \prime}(s) d s=4 \int_{t}^{+\infty} \delta(s) d s .
$$

Hence, we obtain (5.6), concluding the proof of Lemma 5.3.

\subsection{Radial solutions}

Assume that $u$ is radial, satisfies (5.1) and (5.2), and that it is globally defined for positive time. We will show that $u$ has finite variance, which will yield Proposition 5.1 in the case of radial solutions also.

Let $\varphi$ be a radial function such that

$$
0 \leq \varphi(r), \quad \varphi^{\prime \prime}(r) \leq 2, \quad 0 \leq r \leq 1 \Longrightarrow \varphi(r)=r^{2}, \quad 2 \leq r \Longrightarrow \varphi(r)=0 .
$$

Consider the localized variance

$$
y_{R}(t)=\int R^{2} \varphi\left(\frac{x}{R}\right)|u(x, t)|^{2} d x
$$


We know that (5.1) implies $8 \int|\nabla u|^{2}-6 \int|u|^{4}=4\left(\|\nabla Q\|_{2}^{2}-\|\nabla u\|_{2}^{2}\right)$ (see the computation before (5.8)). By explicit calculations, (1.1), (5.1) and the radiality of $u$ and $\varphi$ we get

$$
\begin{gathered}
y_{R}^{\prime}(t)=2 R \operatorname{Im} \int \bar{u} \nabla \varphi\left(\frac{x}{R}\right) \cdot \nabla u \\
y_{R}^{\prime \prime}(t)=4\left(\int|\nabla Q|^{2}-\int|\nabla u|^{2}\right)+A_{R}(u(t))=-4 \delta(t)+A_{R}(u(t)),
\end{gathered}
$$

where

$$
\begin{aligned}
A_{R}(u(t))=4 \int & \left(\varphi^{\prime \prime}\left(\frac{x}{R}\right)-2\right)|\nabla u|^{2} \\
& -\int\left(\Delta \varphi\left(\frac{x}{R}\right)-6\right)|u|^{4}-\frac{1}{R^{2}} \int \Delta^{2} \varphi\left(\frac{x}{R}\right)|u|^{2}
\end{aligned}
$$

Step 1. Concavity of $\boldsymbol{y}_{\boldsymbol{R}}$. We first claim

$$
\exists R_{0}>0, \forall R \geq R_{0}, \quad y_{R}^{\prime \prime}(t) \leq-2 \delta(t) .
$$

By (5.17), we must show that there exists $R_{0}>0$ such that for $R \geq R_{0}$,

$$
A_{R}(u(t)) \leq 2 \delta(t)
$$

The proof is close to [8, Claim 4.3] and we will only sketch it.

Using that $e^{i t} Q$ is a solution of (1.1) such that the corresponding $y_{R}(t)$ is constant and the corresponding $\delta(t)$ is identically zero, we get $A_{R}\left(e^{i t} Q\right)=0$.

Recall the parameter $\delta_{0}$ of Section 4 . First assume that $t \in D_{\delta_{1}}$ (i.e., that $\left.\delta(t) \leq \delta_{1}\right)$, where $\delta_{1} \in\left(0, \delta_{0}\right)$ will be chosen later. By Lemma 4.2 , denoting $v=\alpha Q+h$, we get

$$
u(t)=e^{i t}(Q+v(t)), \quad\|v(t)\|_{H^{1}} \leq C \delta(t) .
$$

Noting that $\varphi^{\prime \prime}(x / R)-2=\Delta \varphi(x / R)-6=\Delta^{2} \varphi(x / R)=0$ for $|x| \leq R$, we get

$$
\begin{aligned}
\left|A_{R}(u(t))\right| & =\left|A_{R}(Q+v)-A_{R}(Q)\right| \\
& \leq C \int_{|x| \geq R}\left(Q^{3}|v|+|v|^{4}+|\nabla Q||\nabla v|+|\nabla v|^{2}+Q|v|+|v|^{2}\right) d x .
\end{aligned}
$$

In view of the exponential decay of $Q$, we obtain

$$
\left|A_{R}(u(t))\right| \leq C\left(e^{-c R} \delta(t)+\delta^{2}(t)+\delta^{4}(t)\right),
$$

which shows that there exists $R_{1}>0, \delta_{1}>0$ such that (5.19) holds for $R \geq R_{1}, t \in D_{\delta_{1}}$. 
We now fix such a $\delta_{1}$ and assume $\delta(t) \geq \delta_{1}$. Note that by our assumptions on $\varphi, \int|\nabla u|^{2}\left(\varphi^{\prime \prime}-2\right) \leq 0$. It remains to bound the two other terms. We have

$$
\frac{1}{R^{2}} \int|u|^{2} \Delta^{2} \varphi(x / R) \leq \frac{C}{R^{2}} M[Q] \leq \delta_{1} \leq \delta(t), \text { if } R \geq R_{2}=\sqrt{\frac{C M[Q]}{\delta_{1}}}
$$

Recall that by Strauss Lemma [30], $u(t)$ being radial, it is bounded and

$$
\forall x \in \mathbb{R}^{3} \backslash\{0\}, \quad|u(x, t)| \leq \frac{C}{|x|}\|u(t)\|_{2}^{1 / 2}\|\nabla u(t)\|_{2}^{1 / 2} .
$$

Hence,

$$
\begin{aligned}
\left.\left|\int\right| u\right|^{4}(\Delta \varphi(x / R)-6) \mid & \leq C \int_{|x| \geq R}|u|^{4} \\
& \leq \frac{C}{R^{2}}\|u\|_{2}^{3}\|\nabla u\|_{2} \leq \frac{C}{R^{2}} M[Q]^{3 / 2} \sqrt{\delta(t)+\|\nabla Q\|_{2}^{2}}
\end{aligned}
$$

Using that $\delta(t) \geq \delta_{1}$, we get that there exists a constant $C_{\delta_{1}}$, depending only on $\delta_{1}$ and such that

$$
\left.\left|\int\right| u\right|^{4}(\Delta \varphi(x / R)-6) \mid \leq \frac{C_{\delta_{1}}}{R^{2}} \delta(t) .
$$

If $R \geq R_{3}=\sqrt{C_{\delta_{1}}}$, we get

$$
\left.\left|\int\right| u\right|^{4}(\Delta \varphi(x / R)-6) \mid \leq \delta(t)
$$

By (5.20) and (5.21), we get (5.19) for $R \geq \max \left\{R_{2}, R_{3}\right\}$ in the case $\delta(t) \geq \delta_{1}$ also.

Step 2. Proof of the finite variance of $\boldsymbol{u}_{\mathbf{0}}$. Let us fix $R \geq R_{0}$, where $R_{0}$ is given by Step 1. We first show that for all $t$ in the domain of existence of $u$,

$$
y_{R}^{\prime}(t)>0 \text {. }
$$

If not, using that for all $t, y^{\prime \prime}(t)<0$, there exists $t_{1}, \varepsilon>0$ such that for $t \geq t_{1}, y_{R}^{\prime}(t)<-\varepsilon$, which contradicts the fact that $y$ is positive and that $u$ is globally defined for positive time.

From the fact that $y_{R}^{\prime}$ is positive and decreasing, we deduce that it has a finite limit as $t$ goes to infinity. But then the integral $\int_{0}^{+\infty} y_{R}^{\prime \prime}(t) d t$ is convergent, which by (5.18) implies

$$
\int_{0}^{+\infty} \delta(s) d s<\infty
$$


Thus, there exists a subsequence $t_{n} \rightarrow+\infty$ such that $\delta\left(t_{n}\right) \rightarrow 0$. By Proposition 2.1, extracting if necessary, there exists $\theta_{0} \in \mathbb{R}$ such that $u\left(t_{n}\right) \rightarrow e^{i \theta_{0}} Q$ in $H^{1}$. By (5.22), $y_{R}$ is increasing, and thus,

$$
y_{R}(0)=\int R^{2} \varphi(x / R)\left|u_{0}\right|^{2} \leq \int R^{2} \varphi(x / R)|Q|^{2} .
$$

Letting $R$ go to infinity, we get

$$
\int|x|^{2}\left|u_{0}\right|^{2}<\infty
$$

which shows that we are in the finite variance case, already treated in subsection 5.1.

\section{Convergence to $Q$ in the case $\left\|\nabla u_{0}\right\|_{2}\left\|u_{0}\right\|_{2}<\|\nabla Q\|_{2}\|Q\|_{2}$}

The main purpose of this section is to prove

Proposition 6.1. Consider a solution $u$ of (1.1) such that

$$
M[u]=M[Q], \quad E[u]=E[Q], \quad\left\|\nabla u_{0}\right\|_{2}<\|\nabla Q\|_{2} .
$$

which does not scatter for positive times. Then there exists $\theta_{0} \in \mathbb{R}, x_{0} \in \mathbb{R}^{3}$, $c, C>0$ such that

$$
\left\|u-e^{i t+i \theta_{0}} Q\left(\cdot-x_{0}\right)\right\|_{H^{1}} \leq C e^{-c t} .
$$

We start by proving, in $\S 6.1$ that a solution $u$ of (1.1) satisfying (6.1) is compact in $H^{1}$ up to a translation $x(t)$ in space. In $\S 6.2$ it is shown by a local virial identity, that the parameter $\delta(t)=\left|\|\nabla u\|_{2}^{2}-\|\nabla Q\|_{2}^{2}\right|$ converges to 0 in mean. In $\S 6.3$, combining the results of the earlier subsections $\S 6.1-6.2$, the estimates of Section 4, and a localized virial approach with a spatial control, we conclude the proof of Proposition 6.1. Finally, $\S 6.4$ is dedicated to the behavior of the special solution $Q^{-}$constructed in Proposition 3.2 for negative time, concluding the proof of Theorem 2 .

\subsection{Compactness properties}

Lemma 6.2. Let $u$ be a solution of (1.1) satisfying the assumptions of Proposition 6.1. Then there exists a continuous function $x(t)$ such that

$$
K:=\{u(x+x(t), t), t \in[0,+\infty)\}
$$

has a compact closure in $H^{1}$. 
Sketch of the proof. We only sketch the argument and refer to the proofs of [16, Prop 4.2], [15, Prop 5.4] and [8, Lemma 2.8] for detailed proofs in similar contexts.

It is sufficient to show that for every time-sequence $\tau_{n} \geq 0$, there exists (extracting if necessary) a subsequence $x_{n}$ such that $u\left(x+x_{n}, \tau_{n}\right)$ has a limit in $H^{1}$ (see e.g. [7, Appendix]).

By the nonradial profile decomposition [7, Lemma 2.1], there exist families of profiles $\psi^{j} \in H^{1}$, and of sequences $x_{n}^{j}$ and $t_{n}^{j}$ such that

$$
\begin{gathered}
u\left(x, \tau_{n}\right)=\sum_{j=1}^{N} e^{-i t_{n}^{j} \Delta} \psi^{j}\left(x-x_{n}^{j}\right)+W_{n}^{M}(x), \\
\lim _{M \rightarrow+\infty} \lim _{n \rightarrow+\infty}\left\|e^{i t \Delta} W_{n}^{M}\right\|_{S\left(\dot{H}^{1 / 2}\right)}=0, \\
\lim _{n \rightarrow+\infty}\left|x_{n}^{j}-x_{n}^{k}\right|+\left|t_{n}^{j}-t_{n}^{k}\right|=+\infty .
\end{gathered}
$$

The crucial point is to show that there is exactly one nonzero profile. Indeed, if for all $j, \psi^{j}=0$, then $u$ must scatter by the local Cauchy problem theory for (1.1).

On the other hand, if at least two profiles are nonzero, then by the Pythagorean expansions properties of the profile decomposition (see (2.3) and (2.8) in [7]) there exists $\varepsilon>0$ such that for all $j$,

$$
M\left[\psi^{j}\right] E\left[e^{-i t_{n}^{j} \Delta} \psi^{j}\right] \leq M[Q] E[Q]-\varepsilon,\left\|\psi^{j}\right\|_{2}\left\|\nabla \psi^{j}\right\|_{2} \leq\|Q\|_{2}\|\nabla Q\|_{2}-\varepsilon
$$

Recall that by [15, 7], a solution of (1.1) with initial condition $v_{0} \in H^{1}$ such that $\left\|v_{0}\right\|_{2}\left\|\nabla v_{0}\right\|_{2}<\|Q\|_{2}\|\nabla Q\|_{2}$ scatters as $t \rightarrow \pm \infty$. By the existence of wave operators for equation (1.1), there exists for all $j$ a function $v_{0}^{j}$ in $H^{1}$ such that the corresponding solution $v^{j}$ of (1.1) satisfies

$$
\lim _{n \rightarrow+\infty}\left\|e^{-i t_{n}^{j} \Delta} \psi^{j}-v^{j}\left(t_{n}^{j}\right)\right\|_{H^{1}}=0 .
$$

Using the arguments of the proof of [15, Prop 5.4], one can show, as a consequence of (6.3) and the scattering of $v^{j}$, that for large $n$, the solution $u\left(x, t+\tau_{n}\right)$ of (1.1) is close (for positive times) to the approximate solution $\sum_{j=1}^{N} v^{j}\left(x-x_{n}^{j}, t+t_{n}^{j}\right)$ of (1.1) (where $N$ is large). Therefore, the solution $u$ must also scatter for positive time, which yields a contradiction, showing that there is only one nonzero profile.

As a consequence,

$$
u\left(x, \tau_{n}\right)=e^{-i t_{n}^{1} \Delta} \psi^{1}\left(x-x_{n}^{1}\right)+W_{n}^{1}(x), \quad \lim _{n \rightarrow+\infty}\left\|e^{i t \Delta} W_{n}^{1}\right\|_{S\left(\dot{H}^{1 / 2}\right)}=0 .
$$


Furthermore, $\lim _{n \rightarrow+\infty}\left\|W_{n}^{1}\right\|_{H^{1}}=0$. If not, for some $\varepsilon>0$,

$$
E\left[e^{-i t_{n}^{1} \Delta} \psi^{1}\right] M\left[e^{-i t_{n}^{1} \Delta} \psi^{1}\right] \leq E[Q] M[Q]-\varepsilon,
$$

and one can show by the preceding arguments that $u$ scatters.

It remains to show that $t_{n}^{1}$ is bounded (and thus, converges up to extraction). If not, we may assume that $t_{n}^{1} \rightarrow+\infty$ or $t_{n}^{1} \rightarrow-\infty$. In the first case,

$$
\begin{aligned}
\left\|e^{i t \Delta} u\left(\tau_{n}\right)\right\|_{S\left((-\infty, 0] ; \dot{H}^{1 / 2}\right)}= & \left\|e^{i\left(t-t_{n}^{1}\right) \Delta} \psi^{1}\right\|_{S\left((-\infty, 0] ; \dot{H}^{1 / 2}\right)}+o_{n}(1) \\
& =\left\|e^{i t \Delta} \psi^{1}\right\|_{S\left(\left(-\infty,-t_{n}^{1}\right] ; \dot{H}^{1 / 2}\right)}+o_{n}(1),
\end{aligned}
$$

which goes to 0 as $n$ goes to $\infty$, showing that $u$ scatters for positive time, a contradiction. Similarly, in the second case

$$
\left\|e^{i t \Delta} u\left(\tau_{n}\right)\right\|_{S\left([0,+\infty) ; \dot{H}^{1 / 2}\right)}=\left\|e^{i t \Delta} \psi^{1}\right\|_{S\left(\left[-t_{n}^{1},+\infty\right) ; \dot{H}^{1 / 2}\right)}+o_{n}(1) \underset{n \rightarrow+\infty}{\longrightarrow} 0 .
$$

Thus, $u\left(\tau_{n}\right)$ satisfies the analogue of (2.5) for negative times, which shows that $u$ scatters for negative and (by the analogue of $(2.6)),\|u\|_{S\left(\left(-\infty, \tau_{n}\right] ; \dot{H}^{1 / 2}\right)}$ goes to 0 as $n$ goes to $\infty$. Since $\tau_{n} \geq 0$, we get that $u=0$, contradicting our assumptions.

Let $u$ be a solution of (1.1) satisfying (6.1). Let $x(t)$ be the translation parameter of Lemma 6.2. Consider $\delta_{0}>0$ as in Section 4. The parameters $X(t), \theta(t), \alpha(t)$ are defined for $t \in D_{\delta_{0}}=\left\{t \mid \delta(t)<\delta_{0}\right\}$. By (4.4) and Lemma 4.2, there exists a constant $C_{0}>0$ such that

$$
\forall t \in D_{\delta_{0}}, \quad \int_{|x-X(t)| \leq 1}|\nabla u|^{2}+|u|^{2} \geq \int_{|x| \leq 1}|\nabla Q|^{2}+|Q|^{2}-C_{0} \delta(t) .
$$

Taking a smaller $\delta_{0}$ if necessary, we can assume that the right hand side of the preceding inequality is bounded from below by a strictly positive constant $\varepsilon_{0}$ on $D_{\delta_{0}}$. Thus,

$$
\forall t \in D_{\delta_{0}}, \quad \int_{|x+x(t)-X(t)| \leq 1}|\nabla u(x+x(t))|^{2}+|u(x+x(t))|^{2} \geq \varepsilon_{0}>0 .
$$

By compactness of $\bar{K}$, it follows that $|x(t)-X(t)|$ is bounded on $D_{\delta_{0}}$. As a consequence, we may modify $x(t)$ so that $K$ defined by (6.2) remains precompact in $H^{1}$ and

$$
\forall t \in D_{\delta_{0}}, \quad x(t)=X(t) .
$$

It is classical that one may choose the function $x$ to be continuous (see [16, Remark 5.4] and [7, Lemma A.3]). Therefore, we have shown 
Corollary 6.3. Let $u$ be as in Proposition 6.1. Then there exists a continuous function $x(t)$ such that (6.5) holds and the set $K$ defined by (6.2) has compact closure in $H^{1}$.

We will also need the following:

Lemma 6.4. Let $u$ be as in Proposition 6.1, and $x(t)$ be defined by Corollary 6.3. Then

$$
P[u]=\operatorname{Im} \int \bar{u} \nabla u d x=0
$$

Furthermore,

$$
\lim _{t \rightarrow+\infty} \frac{x(t)}{t}=0
$$

Proof. Assume $P[u] \neq 0$ and consider, as in [7, Prop. 4.1], the Galilean transformation of $u, w(x, t)=e^{i x \cdot \xi_{0}} e^{-i t\left|\xi_{0}\right|^{2}} u\left(x-2 \xi_{0} t, t\right)$. In order to minimize $E[w]$, we take $\xi_{0}=-P[u] / M[u]$. Then $M[w]=M[u]=M[Q]$, and by the choice of $\xi_{0}, E[w]<E[u]=E[Q]$. By the result of [7], $u$ scatters in $H^{1}$ which contradicts our assumptions, showing (6.6).

For the proof of (6.7) see [7, Lemma 5.1].

\subsection{Convergence in mean}

Lemma 6.5. Let $u$ be a solution of (1.1) satisfying the assumptions of Proposition 6.1. Then

$$
\lim _{T \rightarrow+\infty} \frac{1}{T} \int_{0}^{T} \delta(t) d t=0
$$

where as in Section $4, \delta(t)=\left|\|\nabla Q\|_{2}^{2}-\|\nabla u(t)\|_{2}^{2}\right|$.

As an immediate corollary we get

Corollary 6.6. Under the assumptions of Proposition 6.1, there exists a sequence $t_{n}$ such that $t_{n} \rightarrow+\infty$ and

$$
\lim _{n \rightarrow+\infty} \delta\left(t_{n}\right)=0
$$

In the sequel we will assume, extracting if necessary, that for all $n$, $1+t_{n} \leq t_{n+1}$. 
Proof of Lemma 6.5. Let $\varphi$ be a $C^{\infty}$ positive radial function on $\mathbb{R}^{3}$ such that $\varphi(x)=|x|^{2}$ if $|x| \leq 1$ and $\varphi(x)=0$ if $|x| \geq 2$. Consider the localized variance

$$
y_{R}(t)=\int_{\mathbb{R}^{3}} R^{2} \varphi\left(\frac{x}{R}\right)|u(x, t)|^{2} d x .
$$

Then by explicit computations and (1.1),

$$
y_{R}^{\prime}(t)=2 R \operatorname{Im} \int \bar{u} \nabla \varphi\left(\frac{x}{R}\right) \cdot \nabla u, \quad\left|y_{R}^{\prime}(t)\right| \leq C R .
$$

Furthermore, $y_{R}^{\prime \prime}(t)=\left(8 \int|\nabla u|^{2}-6 \int|u|^{4}\right)+A_{R}(u(t))$, where

$$
\begin{aligned}
A_{R}(u(t)):=4 \sum_{j \neq k} \int \frac{\partial^{2} \varphi}{\partial x_{j} \partial x_{k}}\left(\frac{x}{R}\right) \frac{\partial u}{\partial x_{j}} \frac{\partial \bar{u}}{\partial x_{k}} \\
+4 \sum_{j} \int\left(\frac{\partial^{2} \varphi}{\partial x_{j}^{2}}\left(\frac{x}{R}\right)-2\right)\left|\partial_{x_{j}} u\right|^{2} \\
\quad-\frac{1}{R^{2}} \int \Delta^{2} \varphi\left(\frac{x}{R}\right)|u|^{2}-\int\left(\Delta \varphi\left(\frac{x}{R}\right)-6\right)|u|^{4} .
\end{aligned}
$$

Using as in the proof of Lemma 5.3, that $E[u]=E[Q]$ and $M[u]=M[Q]$, we get

$$
y_{R}^{\prime \prime}(t)=4 \delta(t)+A_{R}(u(t)) .
$$

Note that if $|y| \leq 1,\left(\Delta^{2} \varphi\right)(y)=0, \partial_{x_{j}}^{2} \varphi(y)=2$ and $\Delta \varphi(y)=6$. Thus,

$$
\left|A_{R}(u(t))\right| \leq C \int_{|x| \geq R}|\nabla u|^{2}+|u|^{4}+\frac{1}{R^{2}}|u|^{2} .
$$

Let $x(t)$ be as in Corollary 6.3 and $K$ be defined by (6.2). Let $\varepsilon>0$. By compactness of $K$, there exists $R_{0}(\varepsilon)>0$ such that

$$
\forall t \geq 0, \quad \int_{|x-x(t)| \geq R_{0}(\varepsilon)}|\nabla u|^{2}+|u|^{2}+|u|^{4} \leq \varepsilon .
$$

Furthermore, by (6.7), there exists $t_{0}(\varepsilon) \geq 0$ such that

$$
\forall t \geq t_{0}(\varepsilon), \quad|x(t)| \leq \varepsilon t .
$$

Let

$$
T \geq t_{0}(\varepsilon), \quad R:=\varepsilon T+R_{0}(\varepsilon)+1, \quad t \in\left[t_{0}(\varepsilon), T\right] .
$$


Let us bound the terms in (6.13). Using that $|x(t)| \leq \varepsilon T$ and $R_{0}(\varepsilon)+\varepsilon T \leq R$, we get

$$
\begin{aligned}
\int_{|x| \geq R}|\nabla u|^{2}+|u|^{4}+\frac{1}{R^{2}}|u|^{2} & \leq \int_{|x-x(t)|+|x(t)| \geq R}|\nabla u|^{2}+|u|^{4}+|u|^{2} \\
& \leq \int_{|x-x(t)| \geq R_{0}(\varepsilon)}|\nabla u|^{2}+|u|^{4}+|u|^{2} \leq \varepsilon .
\end{aligned}
$$

By (6.10) and (6.12), we obtain

$$
\int_{t_{0}(\varepsilon)}^{T}\left[4 \delta(t)+A_{R}(u(t))\right] d t=\int_{t_{0}(\varepsilon)}^{T} y_{R}^{\prime \prime}(t) d t \leq\left|y_{R}^{\prime}(T)\right|+\left|y_{R}^{\prime}\left(t_{0}(\varepsilon)\right)\right| \leq C R .
$$

Thus, by (6.13) and (6.16), we have, for some constant $\widetilde{C}>0$, independent of $T$ and $\varepsilon$,

$$
\int_{t_{0}(\varepsilon)}^{T} \delta(t) d t \leq C(R+T \varepsilon) \leq \widetilde{C}\left(R_{0}(\varepsilon)+1+\varepsilon T\right) .
$$

Hence,

$$
\frac{1}{T} \int_{0}^{T} \delta(t) d t \leq \frac{1}{T} \int_{0}^{t_{0}(\varepsilon)} \delta(t) d t+\frac{\widetilde{C}}{T}\left(R_{0}(\varepsilon)+1\right)+\widetilde{C} \varepsilon
$$

Passing to the limit superior as $T \rightarrow+\infty$, then letting $\varepsilon$ tends to 0 , we get (6.8).

\subsection{Exponential convergence}

In this section we prove Proposition 6.1. We refer to [8, Subsection 3.3] and [9, Subsection 3.3] for similar arguments.

The two ingredients of the proof of Proposition 6.1 are the localized virial argument (Lemma 6.7) and a precise control of the variations of the parameter $x(t)$ (Lemma 6.8).

Lemma 6.7. Let $u$ be a solution of (1.1) satisfying the assumptions of Proposition 6.1, and $x(t)$ be as in Corollary 6.3. Then there exists a constant $C$ such that if $0 \leq \sigma<\tau$

$$
\int_{\sigma}^{\tau} \delta(t) d t \leq C\left[1+\sup _{\sigma \leq t \leq \tau}|x(t)|\right](\delta(\sigma)+\delta(\tau)) .
$$

Proof. Consider the localized variance $y_{R}(t)$ defined by (6.9). By (6.10) and (6.12)

$$
y_{R}^{\prime}(t)=2 R \operatorname{Im} \int \bar{u} \nabla \varphi\left(\frac{x}{R}\right) \cdot \nabla u, \quad y_{R}^{\prime \prime}(t)=4 \delta(t)+A_{R}(u(t)),
$$

where $A_{R}$ is defined in (6.11). 
Step 1. Bound on $A_{R}$. We claim that if $\varepsilon>0$, there exists a constant $R_{\varepsilon}$ such that

$$
\forall t \geq 0, \quad|x| \geq R_{\varepsilon}(1+|x(t)|) \Longrightarrow\left|A_{R}(u(t))\right| \leq \varepsilon \delta(t) .
$$

To prove (6.19), we distinguish two cases.

In the case when $\delta(t)$ is small, we use the estimates from Section 4 . Consider $\delta_{0}>0$ as in Section 4 (such that the parameters $\theta(t), X(t), \alpha(t)$ are well-defined for $t \in D_{\delta_{0}}$ ). Let $\delta_{1}$ to be specified later and such that $0<\delta_{1}<\delta_{0}$. Assume that $t \in D_{\delta_{1}}$. Let $v=h+\alpha Q$, then from (4.4) and Lemma 4.2 , we get

$$
\begin{aligned}
& u(x, t)=e^{i(t+\theta(t))}[Q(x-X(t))+v(x-X(t), t)] \\
& \text { and }\|v(t)\|_{H^{1}} \leq C \delta(t) .
\end{aligned}
$$

Note that if $\theta_{0}$ and $X_{0}$ are fixed, then $e^{i \theta_{0}} e^{i t} Q\left(\cdot+X_{0}\right)$ is a solution of (1.1) such that the corresponding $y_{R}(t)$ does not depend on $t$ and also $\delta(t)=0$.

As a consequence, $A_{R}\left(e^{i \theta_{0}} e^{i t} Q\left(\cdot+X_{0}\right)\right)=0$ for any $R$ and $t$. By the definition (6.11) of $A_{R}$ with the change of variables $y=x-X(t)$, we obtain

$$
\begin{aligned}
&\left|A_{R}(u)\right|=\left|A_{R}(u)-A_{R}\left(e^{i(t+\theta(t))} Q(x-X(t))\right)\right| \\
& \leq C \int_{|y+X(t)| \geq R}\left(|\nabla Q(y)||\nabla v(y)|+|\nabla v(y)|^{2}\right.\left.+Q(y)|v(y)|+|v(y)|^{2}+|v(y)|^{4}\right) d y \\
& \leq C\left[\int_{|y+X(t)| \geq R} e^{-|y|}\left(|\nabla v(y)|+|v(y)|+|v(y)|^{3}\right)\right. \\
&\left.\quad+\int_{|y+X(t)| \geq R}\left(|\nabla v(y)|^{2}+|v(y)|^{2}+|v(y)|^{4}\right)\right] .
\end{aligned}
$$

By Lemma 4.2, $\|v(t)\|_{H^{1}} \leq C \delta(t)$, and hence, for some constant $C_{0}>0$, we get

(6.21) $R \geq R_{0}+|X(t)| \Longrightarrow\left|A_{R}(u(t))\right| \leq C_{0}\left[e^{-R_{0}}\left(\delta(t)+\delta(t)^{3}\right)+\delta(t)^{2}+\delta(t)^{4}\right]$.

Choosing $R_{0}$ and $\delta_{1}$ such that $C_{0} e^{-R_{0}} \leq \frac{\varepsilon}{2}$ and $C_{0}\left(e^{-R_{0}} \delta_{1}^{2}+\delta_{1}+\delta_{1}^{3}\right) \leq \frac{\varepsilon}{2}$, we get

$$
R \geq R_{0}+|X(t)| \text { and } \delta(t) \leq \delta_{1} \Longrightarrow\left|A_{R}(u(t))\right| \leq \varepsilon \delta(t) .
$$

Finally, by (6.5) $x(t)=X(t)$ on $D_{\delta_{0}}$, which shows that (6.22) implies (6.19) for $\delta(t)<\delta_{1}$. 
Now assume that $\delta(t) \geq \delta_{1}$. Then by (6.11), there exists a constant $C>0$ such that

$$
\begin{aligned}
\forall t \geq 0, \quad\left|A_{R}(u(t))\right| & \leq C \int_{|x| \geq R}\left(|\nabla u(t)|^{2}+|u(t)|^{4}+|u(t)|^{2}\right) \\
& \leq C \int_{|x-x(t)| \geq R-|x(t)|}\left(|\nabla u(t)|^{2}+|u(t)|^{4}+|u(t)|^{2}\right) .
\end{aligned}
$$

By the compactness of $K$, there exists $R_{1}>0$ such that

$$
R \geq|x(t)|+R_{1} \text { and } \delta(t) \geq \delta_{1} \Longrightarrow\left|A_{R}(u(t))\right| \leq \varepsilon \delta_{1} \leq \varepsilon \delta(t),
$$

hence, (6.19) for $\delta(t) \geq \delta_{1}$, which completes Step 1.

Step 2. End of the proof. By (6.18) and (6.19), we get that there exists $R_{2}>0$ such that

$$
R \geq R_{2}(1+|x(t)|) \Longrightarrow y_{R}^{\prime \prime}(t) \geq 2 \delta(t)
$$

Let $R=R_{2}\left(1+\sup _{\sigma \leq t \leq \tau}|x(t)|\right)$. Then

$$
2 \int_{\sigma}^{\tau} \delta(t) d t \leq \int_{\sigma}^{\tau} y_{R}^{\prime \prime}(t) d t=y_{R}^{\prime}(\tau)-y_{R}^{\prime}(\sigma)
$$

Note that if $\delta(t)<\delta_{0}$, then by (6.18), (6.20) and the change of variables $\xi=x-X(t)$, we get

$$
\begin{aligned}
y_{R}^{\prime}(t)=2 R & \operatorname{Im} \int \bar{v}(\xi) \nabla \varphi\left(\frac{\xi+X(t)}{R}\right) \cdot \nabla Q(\xi) \\
& +2 R \operatorname{Im} \int Q(\xi) \nabla \varphi\left(\frac{\xi+X(t)}{R}\right) \cdot \nabla v(\xi) \\
& +2 R \operatorname{Im} \int \bar{v}(\xi) \nabla \varphi\left(\frac{\xi+X(t)}{R}\right) \cdot \nabla v(\xi),
\end{aligned}
$$

which yields, by Lemma $4.2,\left|y_{R}^{\prime}(t)\right| \leq C R\left(\delta(t)+\delta(t)^{2}\right) \leq C R \delta(t)$. This inequality remains valid if $\delta(t) \geq \delta_{0}$ by the straightforward estimate $\left|y_{R}(t)\right| \leq$ $C R\|\nabla u(t)\|_{2}\|u(t)\|_{2}$. In view of (6.24), we get

$$
\int_{\sigma}^{\tau} \delta(t) d t \leq C R(\delta(\sigma)+\delta(\tau)) \leq C R_{2}\left(1+\sup _{\sigma \leq t \leq \tau}|x(t)|\right)(\delta(\sigma)+\delta(\tau))
$$

which concludes the proof of Lemma 6.7 . 
Lemma 6.8 (Control of the variations of $x(t)$ ). There exists a constant $C>0$ such that

$$
\forall \sigma, \tau>0 \quad \text { with } \quad \sigma+1 \leq \tau, \quad|x(\tau)-x(\sigma)| \leq C \int_{\sigma}^{\tau} \delta(t) d t .
$$

Proof. The proof is very similar to the one in [9, Lemma 3.10]. We sketch it for the sake of completeness. Let $\delta_{0}>0$ be as in Section 4. Let us first show that there exist $\delta_{2}>0$ such that

$$
\forall \tau \geq 0, \quad \inf _{t \in[\tau, \tau+2]} \delta(t) \geq \delta_{2} \quad \text { or } \quad \sup _{t \in[\tau, \tau+2]} \delta(t)<\delta_{0}
$$

If not, there exist two sequences $t_{n}, t_{n}^{\prime} \geq 0$ such that

$$
\delta\left(t_{n}\right) \underset{n \rightarrow+\infty}{\longrightarrow} 0, \quad \delta\left(t_{n}^{\prime}\right) \geq \delta_{0}, \quad\left|t_{n}-t_{n}^{\prime}\right| \leq 2 .
$$

Extracting if necessary, we may assume

$$
\lim _{n \rightarrow+\infty} t_{n}^{\prime}-t_{n}=\tau \in[-2,2] .
$$

By the compactness of $K, u\left(t_{n}, \cdot+x\left(t_{n}\right)\right)$ converges in $H^{1}$ to some $v_{0} \in H^{1}$. By assumption (6.1) and the fact that $\delta\left(t_{n}\right)$ tends to $0, E\left[v_{0}\right]=E[Q]$, $M\left[v_{0}\right]=M[Q]$ and $\left\|\nabla v_{0}\right\|_{2}=\|\nabla Q\|_{2}$. By Proposition 2.1, $v_{0}=e^{i \theta_{0}} Q\left(\cdot-x_{0}\right)$ for some parameters $\theta_{0} \in \mathbb{R}, x_{0} \in \mathbb{R}^{3}$. As a consequence, the solution of (1.1) with the initial condition $v_{0}$ is $e^{i\left(t+\theta_{0}\right)} Q\left(\cdot-x_{0}\right)$. By continuity of flow and (6.27), $u\left(t_{n}^{\prime}, \cdot+x\left(t_{n}\right)\right)$ tends to $e^{i\left(\tau+\theta_{0}\right)} Q\left(\cdot-x_{0}\right)$ in $H^{1}$, which contradicts the fact that $\delta\left(t_{n}^{\prime}\right) \geq \delta_{0}$, completing the proof of (6.26).

We now show (6.25) with the additional condition that $\tau \leq \sigma+2$. By (6.26), we may assume that $\sup _{t \in[\sigma, \tau]} \delta(t)<\delta_{0}$ or $\inf _{t \in[\sigma, \tau]} \delta(t) \geq \delta_{2}$. In the first case, recalling that by the assumption $(6.5), x(t)=X(t)$ on $D_{\delta_{0}}$, we get (6.25) by time-integration of the estimate $\left|X^{\prime}(t)\right| \leq C \delta(t)$ of Lemma 4.3. In the second case, we have $\int_{\sigma}^{\tau} \delta(t) \geq \delta_{2}$ and (6.26) follows from

$$
\exists C>0, \forall s, t \geq 0, \quad|t-s| \leq 2 \Longrightarrow|X(t)-X(s)| \leq C,
$$

which is a straightforward consequence of the compactness of $K$ in $H^{1}$ and the continuity of the flow of equation (1.1).

To complete the proof of Lemma 6.8 , it remains to divide $[\sigma, \tau]$ into intervals of length at least 1 and at most 2 and stick together the previous inequalities to get (4.3) without the assumption $\tau \leq \sigma+2$.

We are now ready to prove Proposition 6.1. Let us first show that $x(t)$ is bounded. 
Consider the sequence $\left\{t_{n}\right\}_{n}$ given by Corollary 6.6. Recall that $t_{n}$ goes to infinity, that $1+t_{n} \leq t_{n+1}$, and that $\delta\left(t_{n}\right)$ tends to 0 . By Lemma 6.7 and Lemma 6.8, there exists a constant $C_{0}>0$ such that if $N<n$,

$$
1+t_{N} \leq t \leq t_{n} \Longrightarrow\left|x\left(t_{N}\right)-x(t)\right| \leq C_{0}\left(1+\sup _{t_{N} \leq s \leq t_{n}}|x(s)|\right)\left[\delta\left(t_{N}\right)+\delta\left(t_{n}\right)\right] .
$$

Choosing $t \in\left[t_{N}+1, t_{n}\right]$ such that $|x(t)|=\sup _{t_{N}+1 \leq s \leq t_{n}}|x(s)|$, we get

$$
\sup _{t_{N}+1 \leq s \leq t_{n}}|x(s)| \leq C(N)+C_{0}\left(1+\sup _{t_{N}+1 \leq s \leq t_{n}}|x(s)|\right)\left[\delta\left(t_{N}\right)+\delta\left(t_{n}\right)\right]
$$

where $C(N)=\left|x\left(t_{N}\right)\right|+C_{0} \sup _{t \in\left[t_{N}, t_{N}+1\right]}|x(t)|$ (we assumed $\delta\left(t_{N}\right)+\delta\left(t_{n}\right) \leq 1$ ). Fix $N$ large enough such that $C_{0} \delta\left(t_{N}\right) \leq \frac{1}{2}$. Then as soon as $t_{n} \geq t_{N}+1$,

$$
\frac{1}{2} \sup _{t_{N}+1 \leq s \leq t_{n}}|x(s)| \leq C(N)+\frac{1}{2}+C_{0}\left(1+\sup _{t_{N}+1 \leq s \leq t_{n}}|x(s)|\right) \delta\left(t_{n}\right) .
$$

Letting $n$ tend to infinity and using again that $\delta\left(t_{n}\right)$ tends to 0 , we get that $|x(t)|$ is bounded on $\left[t_{N}+1,+\infty\right)$, and thus, by continuity, on $[0,+\infty)$.

We will now show that

$$
\exists c, C>0, \forall \sigma \geq 0, \quad \int_{\sigma}^{\infty} \delta(t) d t \leq C e^{-c \sigma}
$$

which will yield, together with Lemma 4.4, the conclusion of Proposition 6.1.

By Lemma 6.7 and the boundedness of $x(t)$,

$$
\forall \sigma, \tau>0 \quad \text { such that } \quad 0 \leq \sigma \leq \tau, \quad \int_{\sigma}^{\tau} \delta(t) d t \leq C(\delta(\sigma)+\delta(\tau)) .
$$

Fix $\sigma$ and take $\tau=t_{n}$, where the sequence $\left(t_{n}\right)_{n}$, given by Corollary 6.6 is such that $\lim _{n} \delta\left(t_{n}\right)=0$. Letting $n$ tend to $\infty$, we get that $\int_{0}^{+\infty} \delta(t) d t$ is finite and for $\sigma \geq 0, \int_{\sigma}^{+\infty} \delta(t) d t \leq C \delta(\sigma)$. Gronwall's Lemma yields (6.28), concluding the proof of Proposition 6.1.

\subsection{Scattering of $Q^{-}$for negative times}

In this paragraph, we conclude the proof of Theorem 2 by showing by contradiction that the special solution $Q^{-}$constructed in Proposition 3.1 and Remark 3.2 scatters as $t \rightarrow-\infty$.

If not, applying the arguments of $\S 6.1-6.3$ to the solutions $Q^{-}$and $t \rightarrow$ $\overline{Q^{-}}(x,-t)$ of $(1.1)$, we get that there exists a parameter $x(t)$, defined for $t \in \mathbb{R}$ and such that $\widetilde{K}=\left\{Q^{-}(\cdot+x(t), t), t \in \mathbb{R}\right\}$ has compact closure 
in $H^{1}$. By the argument at the end of $\S 6.3, x(t)$ is bounded and $\delta(t)$ tends to 0 as $t \rightarrow \pm \infty$. A simple adjustment of Lemma 6.7 gives

$$
\begin{aligned}
-\infty & <\sigma \leq \tau<+\infty \\
\Longrightarrow & \int_{\sigma}^{\tau} \delta(t) d t \leq C\left[1+\sup _{\sigma \leq t \leq \tau}|x(t)|\right](\delta(\sigma)+\delta(\tau)) \leq C(\delta(\sigma)+\delta(\tau)) .
\end{aligned}
$$

Letting $\sigma$ go to $-\infty$ and $\tau$ to $+\infty$, we get $\int_{\mathbb{R}} \delta(t) d t=0$, thus, $\delta(t)=0$ for all $t$, contradicting the assumption $\left\|\nabla u_{0}\right\|_{2}<\|\nabla Q\|_{2}$.

\section{Uniqueness}

In this section, to conclude the proof of Theorem 3, we show the following uniqueness statement:

Proposition 7.1. Let $u$ be a solution of (1.1), defined on $\left[t_{0},+\infty\right)$, such that $E[u]=E[Q], M[u]=M[Q]$ and

$$
\exists c, C>0: \quad\left\|u-e^{i t} Q\right\|_{H^{1}} \leq C e^{-c t} \quad \forall t \geq t_{0} .
$$

Then there exists $A \in \mathbb{R}$ such that $u=U^{A}$, where $U^{A}$ is the solution of (1.1) defined in Proposition 3.1.

The proof of Proposition 7.1 relies on a careful analysis of solutions of the linearized equation (equation (7.2) below), that decay exponentially as $t$ tends to $+\infty$. This analysis, carried out in $\S 7.1$, relies on the spectral properties of $\mathcal{L}$ described in $\S 2.4$. In $\S 7.2$ we finish the proof of Proposition 7.1, and in $\S 7.3$ we gather the results of Sections 5, 6 and 7 to show Theorem 3 .

\subsection{Exponentially small solutions of the linearized equation}

Recall the notation of Section 3, in particular the operator $\mathcal{L}$ and its eigenvalues and eigenfunctions. Consider

$$
v \in C^{0}\left(\left[t_{0},+\infty\right), H^{1}\right) \quad \text { and } \quad g \in C^{0}\left(\left[t_{0},+\infty\right), L^{2}\right)
$$

such that

$$
\begin{aligned}
\partial_{t} v+\mathcal{L} v=g, \quad(x, t) \in \mathbb{R}^{3} \times\left(t_{0},+\infty\right), \\
\|v(t)\|_{H^{1}} \leq C e^{-\gamma_{1} t}, \quad\|g(t)\|_{H^{1}} \leq C e^{-\gamma_{2} t}, \quad t \geq t_{0},
\end{aligned}
$$

where

$$
0<\gamma_{1}<\gamma_{2}
$$

For any $\gamma \in \mathbb{R}$, denote by $\gamma^{-}$a positive number arbitrary close to $\gamma$ and such that $0<\gamma^{-}<\gamma$.

We now prove the following self-improving estimate. 
Lemma 7.2. Under the above assumptions,

a. if $\gamma_{2} \leq e_{0}$, then $\|v(t)\|_{H^{1}} \leq C e^{-\gamma_{2}^{-} t}$,

b. if $\gamma_{2}>e_{0}$, then there exists $A \in \mathbb{R}$ such that $v(t)=A e^{-e_{0} t} \mathcal{Y}_{+}+w(t)$ with $\|w(t)\|_{H^{1}} \leq C e^{-\gamma_{2}^{-} t}$.

Proof. In this proof we work with the real $L^{2}$-scalar product, denoted by $(\cdot, \cdot)$,

$$
(u, v)=\operatorname{Re} \int u \bar{v}=\int \operatorname{Re} u \operatorname{Re} v+\int \operatorname{Im} u \operatorname{Im} v .
$$

We first normalize the eigenfunctions of $\mathcal{L}$. Denote

$$
Q_{0}:=\frac{i Q}{\|Q\|_{2}}, \quad Q_{j}:=\frac{\partial_{x_{j}} Q}{\left\|\partial_{x_{j}} Q\right\|_{2}} .
$$

From $\S 2.4$ recall the quadratic form on $H^{1}, \Phi$, and its associated bilinear form $B$. From (2.14) we have

$$
\forall j=0, \ldots 3, \quad \forall h \in H^{1}, \quad B\left(Q_{j}, h\right)=0, \quad\left\|Q_{j}\right\|_{2}=1 .
$$

Recall that $\Phi\left(\mathcal{Y}_{+}\right)=\Phi\left(\mathcal{Y}_{-}\right)=0$ and $B\left(\mathcal{Y}_{+}, \mathcal{Y}_{-}\right) \neq 0$. Normalize the eigenfunctions $\mathcal{Y}_{+}, \mathcal{Y}_{-}$such that $B\left(\mathcal{Y}_{+}, \mathcal{Y}_{-}\right)=1$. We have

$$
h \in G_{\perp}^{\prime} \Longleftrightarrow \forall j, \quad\left(Q_{j}, h\right)=0 \text { and } B\left(\mathcal{Y}_{+}, h\right)=B\left(\mathcal{Y}_{-}, h\right)=0 .
$$

Indeed, if $h_{1}=\operatorname{Re} h, h_{2}=\operatorname{Im} h$, in view of (2.13) and Remark 2.4,

$$
B\left(\mathcal{Y}_{+}, h\right)=\frac{e_{0}}{2}\left[\left(\mathcal{Y}_{2}, h_{1}\right)-\left(\mathcal{Y}_{1}, h_{2}\right)\right], \quad B\left(\mathcal{Y}_{-}, h\right)=\frac{e_{0}}{2}\left[\left(\mathcal{Y}_{2}, h_{1}\right)+\left(\mathcal{Y}_{1}, h_{2}\right)\right]
$$

which shows that the orthogonality condition $\left(2.17^{\prime}\right)$ is equivalent to the condition $B\left(\mathcal{Y}_{+}, h\right)=B\left(\mathcal{Y}_{-}, h\right)=0$.

Next, write

$$
\begin{aligned}
& v(t)=\alpha_{+}(t) \mathcal{Y}_{+}+\alpha_{-}(t) \mathcal{Y}_{-}+\sum_{j=0}^{3} \beta_{j}(t) Q_{j}+v_{\perp}(t), \quad v_{\perp} \in G_{\perp}^{\prime}, \\
& \text { where } \alpha_{+}(t)=B\left(v(t), \mathcal{Y}_{-}\right), \quad \alpha_{-}(t)=B\left(v(t), \mathcal{Y}_{+}\right) \\
& \forall j \in\{0, \ldots, 3\}, \quad \beta_{j}(t)=\left(v(t), Q_{j}\right)-\alpha_{+}(t)\left(\mathcal{Y}_{+}, Q_{j}\right)-\alpha_{-}(t)\left(\mathcal{Y}_{-}, Q_{j}\right) .
\end{aligned}
$$

By the radiality of $\mathcal{Y}_{ \pm}$and $Q$, we have $\left(\mathcal{Y}_{+}, Q_{j}\right)=\left(\mathcal{Y}_{-}, Q_{j}\right)=0$ for $j=1,2,3$, but we will not need this property in the sequel. 
Step 1. Differential equations on the coefficients. Let us show

$$
\begin{aligned}
\left|\alpha_{-}^{\prime}(t)-e_{0} \alpha_{-}(t)\right| \leq C e^{-\gamma_{2} t}, \quad\left|\alpha_{+}^{\prime}(t)+e_{0} \alpha_{+}(t)\right| \leq C e^{-\gamma_{2} t} . \\
\forall j \in\{0, \ldots, 3\}, \quad\left|\beta_{j}^{\prime}(t)\right| \leq C\left(\left\|v_{\perp}(t)\right\|_{2}+e^{-\gamma_{2} t}\right) \\
\left|\frac{d}{d t} \Phi(v(t))\right| \leq e^{-\left(\gamma_{1}+\gamma_{2}\right) t}
\end{aligned}
$$

First note that $\mathcal{L}$ is antisymmetric for the bilinear form $B$. Indeed, for $g, h \in H^{2}$

$$
B(g, \mathcal{L} h)=\frac{1}{2}\left(L_{+} g_{1},-L_{-} h_{2}\right)+\frac{1}{2}\left(L_{-} g_{2}, L_{+} h_{1}\right)=-B(\mathcal{L} g, h) .
$$

By (7.2) and (7.5), we have

$$
\begin{aligned}
\alpha_{-}^{\prime}(t) & =B\left(\partial_{t} v, \mathcal{Y}_{+}\right)=B\left(-\mathcal{L} v+g, \mathcal{Y}_{+}\right) \\
& =B\left(v, \mathcal{L} \mathcal{Y}_{+}\right)+B\left(g, \mathcal{Y}_{+}\right)=e_{0} \alpha_{-}(t)+B\left(g, \mathcal{Y}_{+}\right)
\end{aligned}
$$

In view of assumption (7.3) on $g$, we get the inequality on $\alpha_{-}(t)$ in (7.7). The inequality on $\alpha_{+}(t)$ follows from the same argument.

By (7.6), we obtain

$$
\begin{aligned}
\beta_{j}^{\prime} & =\left(\partial_{t} v-\alpha_{+}^{\prime} \mathcal{Y}_{+}-\alpha_{-}^{\prime} \mathcal{Y}_{-}, Q_{j}\right)=\left(-\mathcal{L} v-\alpha_{+}^{\prime} \mathcal{Y}_{+}-\alpha_{-}^{\prime} \mathcal{Y}_{-}, Q_{j}\right)+\left(g, Q_{j}\right) \\
& =\left(-\alpha_{+} \mathcal{L} \mathcal{Y}_{+}-\alpha_{-} \mathcal{L} \mathcal{Y}_{-}-\alpha_{+}^{\prime} \mathcal{Y}_{+}-\alpha_{-}^{\prime} \mathcal{Y}_{-}, Q_{j}\right)-\left(\mathcal{L} v_{\perp}, Q_{j}\right)+\left(g, Q_{j}\right)
\end{aligned}
$$

Applying (7.7), the first term above is estimated as

$$
\left|\left(-\alpha_{+} \mathcal{L} \mathcal{Y}_{+}-\alpha_{-} \mathcal{L} \mathcal{Y}_{-}-\alpha_{+}^{\prime} \mathcal{Y}_{+}-\alpha_{-}^{\prime} \mathcal{Y}_{-}, Q_{j}\right)\right| \leq C e^{-\gamma_{2} t}
$$

The assumption (7.3) implies $\left|\left(g, Q_{j}\right)\right| \leq C e^{-\gamma_{2} t}$. Furthermore, $\left(\mathcal{L} v_{\perp}, Q_{j}\right)=$ $\left(v_{\perp}, \mathcal{L}^{*} Q_{j}\right)$, where $\mathcal{L}^{*}:=\left(\begin{array}{cc}0 & L_{+} \\ -L_{-} & 0\end{array}\right)$ is the $L^{2}$-adjoint of $\mathcal{L}$, which shows the estimate $\left|\left(\mathcal{L} v_{\perp}, Q_{j}\right)\right| \leq C\left\|v_{\perp}\right\|_{2}$, completing the proof of (7.8).

It remains to prove (7.9). We have

$$
\frac{d}{d t} \Phi(v(t))=2 B\left(\partial_{t} v(t), v(t)\right)=-2 B(\mathcal{L} v, v)+2 B(g, v) .
$$

As $B(\mathcal{L} v, v)=-B(\mathcal{L} v, v)$, we get that $B(\mathcal{L} v, v)=0$, which yields (7.9), using again the assumption (7.3) on $g$, and hence, completing Step 1.

Step 2. Let us show

$$
\begin{aligned}
\left|\alpha_{+}(t)\right| & \leq C e^{-\gamma_{2}^{-} t} & & \text { if } \gamma_{2} \leq e_{0}, \\
\exists A \in \mathbb{R}, \quad\left|\alpha_{+}(t)-A e^{-e_{0} t}\right| & \leq C e^{-\gamma_{2} t} & & \text { if } \gamma_{2}>e_{0} .
\end{aligned}
$$


Indeed, by the second inequality in (7.7), we obtain

$$
\left|\frac{d}{d t}\left(e^{e_{0} t} \alpha_{+}(t)\right)\right| \leq C e^{\left(e_{0}-\gamma_{2}\right) t} .
$$

First assume that $\gamma_{2} \leq e_{0}$. Then by (7.12), for $t \geq t_{0}$,

$$
\left|e^{e_{0} t} \alpha_{+}(t)\right| \leq \begin{cases}e^{e_{0} t_{0}} \alpha_{+}\left(t_{0}\right)+C e^{\left(e_{0}-\gamma_{2}\right) t} & \text { if } e_{0}>\gamma_{2} \\ e^{e_{0} t_{0}} \alpha_{+}\left(t_{0}\right)+C\left(t-t_{0}\right) & \text { if } e_{0}=\gamma_{2}\end{cases}
$$

which gives (7.10).

Now assume $\gamma_{2}>e_{0}$. Then $\int_{t_{0}}^{\infty} e^{\left(e_{0}-\gamma_{2}\right) t} d t<\infty$. By $(7.12)$, we get that $e^{e_{0} t} \alpha_{+}(t)$ has a limit $A$ as $t \rightarrow \infty$ and

$$
\left|e^{e_{0} t} \alpha_{+}(t)-A\right| \leq C e^{\left(e_{0}-\gamma_{2}\right) t}
$$

implying (7.11).

Step 3. Conclusion of the proof in a reduced case. Let us conclude the proof when $\gamma_{2} \leq e_{0}$, or when $\gamma_{2}>e_{0}$ and $A=0$. In both cases we have, in view of (7.7), (7.10) and (7.11),

$$
\forall t \geq t_{0}, \quad\left|\alpha_{+}(t)\right|+\left|\alpha_{+}^{\prime}(t)\right| \leq C e^{-\gamma_{2}^{-} t} .
$$

By the first inequality in (7.7),

$$
\left|\frac{d}{d t}\left(e^{-e_{0} t} \alpha_{-}(t)\right)\right| \leq C e^{-\left(e_{0}+\gamma_{2}\right) t}
$$

Integrating between $t$ and $+\infty$, we get $\left|\alpha_{-}(t)\right| \leq C e^{-\gamma_{2} t}$, and by (7.7), it follows that

$$
\forall t \geq t_{0}, \quad\left|\alpha_{-}(t)\right|+\left|\alpha_{-}^{\prime}(t)\right| \leq C e^{-\gamma_{2} t} .
$$

By the decomposition (7.4) of $v$, and recalling that as $v_{\perp} \in G_{\perp}^{\prime}$, we have $B\left(\mathcal{Y}_{+}, v_{\perp}\right)=B\left(\mathcal{Y}_{-}, v_{\perp}\right)=0$, and that $B\left(\mathcal{Y}_{+}, \mathcal{Y}_{-}\right)=1, B\left(\mathcal{Y}_{+}, \mathcal{Y}_{+}\right)=$ $B\left(\mathcal{Y}_{-}, \mathcal{Y}_{-}\right)=0$, we get

$$
\Phi(v)=B(v, v)=B\left(v_{\perp}, v_{\perp}\right)+2 \alpha_{+} \alpha_{-} .
$$

By (7.9), (7.13) and (7.14),

$$
\left|\frac{d}{d t} B\left(v_{\perp}, v_{\perp}\right)\right| \leq C\left(e^{-2 \gamma_{2}^{-} t}+e^{-\left(\gamma_{1}+\gamma_{2}\right) t}\right) \leq C e^{-\left(\gamma_{1}+\gamma_{2}\right) t} .
$$


Noting that $B\left(v_{\perp}, v_{\perp}\right) \rightarrow 0$ as $t \rightarrow+\infty$, and integrating the previous inequality between $t$ and $+\infty$, we get $\left|B\left(v_{\perp}, v_{\perp}\right)\right| \leq C e^{-\left(\gamma_{1}+\gamma_{2}\right) t}$. By Proposition 2.3, we obtain

$$
\forall t \geq t_{0}, \quad\left\|v_{\perp}(t)\right\|_{H^{1}} \leq C e^{-\left(\frac{\gamma_{1}+\gamma_{2}}{2}\right) t} .
$$

Let $j \in\{0, \ldots, 3\}$. By (7.8), and (7.15),

$$
\left|\beta_{j}^{\prime}(t)\right| \leq C\left(e^{-\left(\frac{\gamma_{1}+\gamma_{2}}{2}\right) t}+e^{-\gamma_{2} t}\right) \leq C e^{-\left(\frac{\gamma_{1}+\gamma_{2}}{2}\right) t} .
$$

Integrating again between $t$ and $+\infty$, we get

$$
\forall t \geq t_{0}, \quad\left|\beta_{j}(t)\right| \leq C e^{-\left(\frac{\gamma_{1}+\gamma_{2}}{2}\right) t} .
$$

In view of the decomposition (7.4) of $v$, the inequalities (7.13), (7.14), (7.15) and (7.16) imply

$$
\forall t \geq t_{0}, \quad\|v(t)\|_{H^{1}} \leq C e^{-\left(\frac{\gamma_{1}+\gamma_{2}}{2}\right) t} .
$$

Thus, $v$ and $g$ satisfy the assumptions (7.3), with $\gamma_{1}$ replaced by $\gamma_{1}^{\prime}=\frac{\gamma_{1}+\gamma_{2}}{2}$. An iteration argument yields

$$
\|v(t)\|_{H^{1}} \leq C e^{-\gamma_{2}^{-} t}
$$

which concludes the proof when $\gamma_{2} \leq e_{0}$ or $A=0$.

Step 4. Conclusion of the proof in the case $\gamma_{2}>e_{0}, A \neq 0$. Note that if $\gamma_{1}>e_{0}$, we must have $A=0$, so that we may assume $\gamma_{1} \leq e_{0}$. Let

$$
\tilde{v}(t)=v(t)-A e^{-e_{0} t} \mathcal{Y}_{+} .
$$

Then

$$
\partial_{t} \tilde{v}(t)+\mathcal{L} \tilde{v}(t)=g(t), \quad\|\tilde{v}(t)\|_{H^{1}} \leq C e^{-\gamma_{1} t},
$$

and by (7.11),

$$
\lim _{t \rightarrow+\infty} e^{e_{0} t} \tilde{\alpha}_{+}(t)=0
$$

where $\tilde{\alpha}_{+}(t)=B\left(\tilde{v}(t), \mathcal{Y}_{-}\right)$is the coefficient of $\mathcal{Y}_{+}$in the decomposition of $\tilde{v}(t)$ analogous to (7.4). Thus, $\tilde{v}(t)$ and $g$ satisfy all the assumptions of Step 3. Hence,

$$
\left\|v(t)-A e^{-e_{0} t} \mathcal{Y}_{+}\right\|_{H^{1}} \leq C e^{-\gamma_{2}^{-} t},
$$

which concludes the proof of Lemma 7.2 in this case also. 


\subsection{Uniqueness}

Let us prove Proposition 7.1. Let $u$ satisfy the hypothesis and write $u=$ $e^{i t}(Q+h)$.

Step 1. Improvement of the decay at infinity. We start with showing that if $e_{0}^{-}$is any positive number such that $e_{0}^{-}<e_{0}$,

$$
\forall t \geq t_{0}, \quad\|h(t)\|_{H^{1}} \leq C e^{-e_{0}^{-} t} .
$$

Indeed, we have $\partial_{t} h+\mathcal{L} h=R(h)$, where the remainder term $R(h)$, defined in (2.9), is a sum of quadratic and cubic terms in $h$. By the assumption (7.1), Strichartz estimates and Sobolev embeddings, $\|h(t)\|_{p} \leq C e^{-c t}$ for every $p \in[2, \infty]$, which yields the bound $\|R(h)\|_{H^{1}} \leq C e^{-2 c t}$. Thus, $h$ satisfies the assumptions of Lemma 7.2 with $g=R(h), \gamma_{1}=c, \gamma_{2}=2 c$. If $2 c>e_{0}$, the proof is complete. If not, we get $\|h(t)\|_{H^{1}} \leq C e^{-2 c^{-} t}$, and the result follows from an iteration argument.

Step 2. Consider the special solutions $U^{A}$ constructed in Proposition 3.1, and write $U^{A}=e^{i t}\left(Q+h^{A}\right)$. Let us show that there exists $A \in \mathbb{R}$ such that for all $\gamma>0$,

$$
\exists C>0, \forall t \geq t_{0}, \quad\left\|h(t)-h^{A}(t)\right\|_{H^{1}} \leq C e^{-\gamma t} .
$$

According to Step 1, $h$ fulfills the assumptions of Lemma 7.2 with $\gamma_{1}=e_{0}^{-}$, $\gamma_{2}=2 e_{0}^{-}$. Thus, there exists $A \in \mathbb{R}$ such that

$$
\left\|h(t)-A e^{-e_{0} t} \mathcal{Y}_{+}\right\|_{H^{1}} \leq C e^{-2 e_{0}^{-} t} .
$$

By the asymptotic development of $h^{A}$ obtained in Section 3,

$$
\left\|h^{A}(t)-A e^{-e_{0} t} \mathcal{Y}_{+}\right\|_{H^{1}} \leq C e^{-2 e_{0} t} .
$$

Thus, (7.20) yields (7.19) for any $\gamma<2 e_{0}$. We next show that if (7.19) holds for some $\gamma>e_{0}$, it also holds for $\gamma^{\prime}=\gamma+\frac{1}{2} e_{0}$. Note that $h-h^{A}$ is a solution to the equation

$$
\partial_{t}\left(h-h^{A}\right)+\mathcal{L}\left(h-h^{A}\right)=R(h)-R\left(h^{A}\right) .
$$

By the explicit expression of $R$, and Sobolev inequalities, we get

$$
\left\|R(h)-R\left(h^{A}\right)\right\|_{2} \leq C\left\|h-h^{A}\right\|_{H^{1}}\left(\|h\|_{H^{1}}+\left\|h^{A}\right\|_{H^{1}}+\|h\|_{H^{1}}^{2}+\left\|h^{A}\right\|_{H^{1}}^{2}\right) .
$$

If (7.19) holds for some $\gamma>e_{0}$, then

$$
\left\|R(h)-R\left(h^{A}\right)\right\|_{2} \leq C e^{-\left(e_{0}+\gamma\right) t},
$$

which shows that $h-h^{A}$ fulfills the assumptions of Lemma 7.2 with $\gamma_{1}=\gamma$, $\gamma_{2}=\gamma+e_{0}$, yielding (7.20) with $\gamma+\frac{1}{2} e_{0}$ instead of $\gamma$. Step 2 is complete. 
Step 3. Uniqueness argument. We are now ready to finish the proof of Proposition 7.1. Let $v:=h-h^{A}$. We must show that $v=0$. We will use that $v$ is a solution to the following Schrödinger equation

$$
i \partial_{t} v+\Delta v-v=-2 Q^{2} v-i Q^{2} \bar{v}+M
$$

where $M(t)=i R(h(t))-i R\left(h^{A}(t)\right)$. By Hölder's inequality and the decay of $h$ and $h^{A}$ at infinity, there exists a constant $C_{1}>0$ such that

$$
\forall t \geq t_{0}, \quad\|M(t)\|_{6 / 5} \leq C_{1} e^{-e_{0} t}\|v(t)\|_{2} .
$$

Let $t_{1} \geq t_{0}, \tau>0$ and $I=\left(t_{1}, t_{1}+\tau\right)$. By Strichartz estimates, there exists $K>0$ such that

$$
\|v\|_{L^{\infty}\left(I ; L^{2}\right)} \leq K\left\{\left\|v\left(t_{1}+\tau\right)\right\|_{2}+\left\|Q^{2} v\right\|_{L^{1}\left(I, L^{2}\right)}+\|M(t)\|_{L^{2}\left(I ; L^{6 / 5}\right)}\right\} .
$$

Integrating in time on $I$ the square of (7.22), we get

$$
\|M(t)\|_{L^{2}\left(I, L^{6 / 5}\right)} \leq \frac{C_{1}}{\sqrt{2 e_{0}}} e^{-e_{0} t_{1}}\|v\|_{L^{\infty}\left(I, L^{2}\right)}
$$

Furthermore,

$$
\left\|Q^{2} v\right\|_{L^{1}\left(I, L^{2}\right)} \leq \tau\left\|Q^{2}\right\|_{\infty}\|v\|_{L^{\infty}\left(I, L^{2}\right)} .
$$

Hence,

$$
\|v\|_{L^{\infty}\left(I ; L^{2}\right)} \leq K\left\{\left\|v\left(t_{1}+\tau\right)\right\|_{2}+\left(\tau\left\|Q^{2}\right\|_{\infty}+\frac{C_{1}}{\sqrt{2 e_{0}}} e^{-e_{0} t_{1}}\right)\|v\|_{L^{\infty}\left(I, L^{2}\right)}\right\} .
$$

Let $\tau=\frac{1}{3 K\left\|Q^{2}\right\|_{\infty}}$, choose $T \geq t_{0}$ such that $\frac{C_{1}}{\sqrt{2 e_{0}}} e^{-e_{0} T} \leq \frac{1}{3 K}$. Then for $t_{1} \geq T$,

$$
\left\|v\left(t_{1}\right)\right\|_{2} \leq\|v\|_{L^{\infty}\left(I ; L^{2}\right)} \leq 3 K\left\|v\left(t_{1}+\tau\right)\right\|_{2} .
$$

By induction we get

$$
\left.(3 K)^{n}\|v(T)\|_{2} \leq \| v(T+n \tau)\right) \|_{2},
$$

which contradicts (7.19) if $\gamma$ is large enough, unless $v(T)=0$. Thus, $h(T)=$ $h^{A}(T)$ and by uniqueness in (1.1), $h=h^{A}$, and thus, $u=U^{A}$, concluding the proof of Proposition 7.1. 


\subsection{Proof of the classification result}

In this subsection we prove Theorem 3 .

We first show that if $A \neq 0, U^{A}$ is equal to $Q^{+}$(if $A>0$ ) or $Q^{-}$(if $A<0)$ up to a translation in time and a multiplication by a complex number of modulus 1. Indeed, by (3.1) and the definition of $Q^{ \pm}$in Remark 3.2, we have

$$
Q^{ \pm}(t)=e^{i t} Q \pm e^{-e_{0} t_{0}} e^{\left(i-e_{0}\right) t} \mathcal{Y}_{+}+O\left(e^{-2 e_{0} t}\right) \text { in } H^{1}
$$

Fix $A>0$ (the proof is similar when $A<0$ ). Let $t_{1}=-t_{0}-\frac{1}{e_{0}} \log A$, so that $e^{-e_{0}\left(t_{0}+t_{1}\right)}=A$. By (3.1) and (7.23), we obtain

$$
\begin{aligned}
e^{-i t_{1}} Q^{+}\left(t+t_{1}\right)=e^{i t} Q+e^{-e_{0}\left(t_{0}+t_{1}\right)} e^{-e_{0} t} e^{i t} \mathcal{Y}_{+}+O\left(e^{-2 e_{0} t}\right) \\
=U^{A}+O\left(e^{-2 e_{0} t}\right) \text { in } H^{1}
\end{aligned}
$$

As a consequence $e^{-i t_{1}} Q^{+}\left(t+t_{1}\right)-e^{i t} Q$ tends to 0 exponentially in $H^{1}$ as $t \rightarrow+\infty$. By Proposition 7.1, there exists $\widetilde{A}$ such that $e^{-i t_{1}} Q^{+}\left(t+t_{1}\right)=U^{\widetilde{A}}$. By (7.24) we have $\widetilde{A}=A$, which shows that $U^{A}=e^{-i t_{1}} Q^{+}\left(t+t_{1}\right)$.

Let $u$ be a solution of (1.1) satisfying the assumptions of Theorem 3 . Then $M[u] E[u]=M[Q] E[Q]$. Rescaling $u$ we may assume

$$
M[u]=M[Q], \quad E[u]=E[Q] .
$$

If $\left\|\nabla u_{0}\right\|_{2}=\|\nabla Q\|_{2}$ (case (b)), then by the variational characterization of $Q$ (see $\S 2.1) u_{0}(x)=e^{i \theta_{0}} Q\left(x-x_{0}\right)$ for some parameters $\theta_{0}, x_{0}$, and thus, by uniqueness of the Cauchy problem (1.1), $u(x, t)=e^{i \theta_{0}+i t} Q\left(x-x_{0}\right)$. Thus, $u$ is equal to $e^{i t} Q$ up to the symmetries of the equation, yielding case (b).

Assume next $\left\|\nabla u_{0}\right\|_{2}<\|\nabla Q\|_{2}$ (case (a)). By assumption, $u$ does not scatter for both positive and negative times. Replacing $u(x, t)$ by $\bar{u}(x,-t)$ if necessary, we may assume that $u$ does not scatter for positive times. By Proposition 6.1, there exists $\theta_{0} \in \mathbb{R}, x_{0} \in \mathbb{R}^{3}$, and $c, C>0$ such that

$$
\left\|u(t)-e^{i t+i \theta_{0}} Q\left(\cdot-x_{0}\right)\right\|_{H^{1}} \leq C e^{-c t}, \quad t>0 .
$$

Hence, $v(x, t)=e^{-i \theta_{0}} u\left(x+x_{0}, t\right)$ satisfies the assumptions of Proposition 7.1, which shows that $v=U^{A}$ for some $A$. As $\left\|\nabla u_{0}\right\|_{2}<\|\nabla Q\|_{2}$, the parameter $A$ must be negative proving that $v$ (and thus $u$ ) is equal to $Q^{-}$up to the symmetries of the equation. Therefore, case (a) of the theorem follows.

The proof of case (c), combining Proposition 5.1 and Proposition 7.1, is similar to the proof of case (a) and left to the reader. 


\section{A. Coercivity properties of the quadratic form}

This appendix is dedicated to the proof of the results of $\S 2.4$. Before proving Proposition 2.7, we show (2.12). Consider $h \in H^{1}$ and assume $E[Q+h]=$ $E[Q]$ and $M[Q+h]=M[Q]$. Expanding $E[Q+h]$ in terms of $Q$ and $h$, we get

$$
\begin{aligned}
E[Q+h]=E[Q] & +\int \nabla Q \cdot \nabla h_{1}-\int Q^{3} h_{1}+\frac{1}{2} \int|\nabla h|^{2} \\
& -\frac{1}{2} \int Q^{2}\left(3 h_{1}^{2}+h_{2}^{2}\right)-\int Q|h|^{2} h_{1}-\frac{1}{4} \int|h|^{4} .
\end{aligned}
$$

Since $E[Q+h]=E[Q]$ and

$$
\int \nabla Q \cdot \nabla h_{1}-\int Q^{3} h_{1}=-\int\left(\Delta Q+Q^{3}\right) h_{1}=-\int Q h_{1}
$$

by (2.1), we obtain

$$
0=-\int Q h_{1}+\frac{1}{2} \int|\nabla h|^{2}-\frac{1}{2} \int Q^{2}\left(3 h_{1}^{2}+h_{2}^{2}\right)-\int Q|h|^{2} h_{1}-\frac{1}{4} \int|h|^{4} .
$$

Furthermore, $M[Q+h]=M[Q]$ implies $2 \int Q h_{1}+\int|h|^{2}=0$, yielding

$$
\Phi(h)=\int Q|h|^{2} h_{1}+\frac{1}{4} \int|h|^{4}
$$

which gives (2.12).

The remainder of the Appendix is dedicated to the proof of Proposition 2.7 .

\section{A.1. Coercivity of $\Phi$ on $G_{\perp}$}

Let us prove (2.18) when $h \in G_{\perp}$ (see [33, 34], [31, ex. B11-B14] for similar proofs for mass-subcritical NLS). We divide the proof into two steps.

Step 1. Nonnegativity. We show, as a consequence of Gagliardo-Nirenberg inequality (2.2), that if $h \in H^{1}$ satisfies (2.17), then

$$
\Phi(h) \geq 0
$$

For $u \in H^{1}$, let

$$
I(u):=\frac{\|\nabla u\|_{2}^{3}\|u\|_{2}}{\|\nabla Q\|_{2}^{3}\|Q\|_{2}}-\frac{\|u\|_{4}^{4}}{\|Q\|_{4}^{4}} .
$$


By (2.2), $I(u) \geq 0$. Take $h \in H^{1}, \alpha \in \mathbb{R}$ and compute the expansion of $I(Q+\alpha h)$ in $\alpha$ of order 2. By (2.17), we have $\int \nabla Q \cdot \nabla h_{1}=0$, and thus,

$$
\left(\int|\nabla(Q+\alpha h)|^{2}\right)^{\frac{3}{2}}=\left(\int|\nabla Q|^{2}\right)^{3 / 2}\left(1+\frac{3}{2} \alpha^{2} \frac{\int|\nabla h|^{2}}{\int|\nabla Q|^{2}}+O\left(\alpha^{4}\right)\right) .
$$

Furthermore,

$$
\begin{aligned}
& \left(\int|Q+\alpha h|^{2}\right)^{1 / 2} \\
& \quad=\left(\int Q^{2}\right)^{1 / 2}\left(1+\alpha \frac{\int Q h_{1}}{\int Q^{2}}+\frac{1}{2} \alpha^{2} \frac{\int|h|^{2}}{\int Q^{2}}-\frac{1}{2} \alpha^{2} \frac{\left(\int Q h_{1}\right)^{2}}{\left(\int Q^{2}\right)^{2}}+O\left(\alpha^{3}\right)\right)
\end{aligned}
$$

and

$$
\begin{aligned}
\int \mid Q & +\left.\alpha h\right|^{4} \\
& =\left(\int Q^{4}\right)\left(1+4 \alpha \frac{\int Q^{3} h_{1}}{\int Q^{4}}+\alpha^{2} \frac{\int 6 Q^{2} h_{1}^{2}+\int 2 Q^{2} h_{2}^{2}}{\int Q^{4}}\right)+O\left(\alpha^{3}\right) .
\end{aligned}
$$

Substituting above quantities into (A.3), we obtain that $I(Q+\alpha h)$ is equal to

$$
\begin{aligned}
\left(1+\frac{3}{2} \alpha^{2} \frac{\int|\nabla h|^{2}}{\int|\nabla Q|^{2}}\right) & \left(1+\alpha \frac{\int Q h_{1}}{\int Q^{2}}+\frac{1}{2} \alpha^{2} \frac{\int|h|^{2}}{\int Q^{2}}-\frac{1}{2} \alpha^{2} \frac{\left(\int Q h_{1}\right)^{2}}{\left(\int Q^{2}\right)^{2}}\right) \\
& -\left(1+\alpha \frac{\int 4 Q^{3} h_{1}}{\int Q^{4}}+\alpha^{2} \frac{\int 6 Q^{2} h_{1}^{2}+2 Q^{2} h_{2}^{2}}{\int Q^{4}}\right)+O\left(\alpha^{3}\right) .
\end{aligned}
$$

Since $I(Q)=0$ and $I(Q+\alpha h) \geq 0$ for all real $\alpha$, the linear term in $\alpha$ in the previous estimate is zero, and the quadratic term is nonnegative. Applying $\int|\nabla Q|^{2}=3 \int Q^{2}$ and $\int Q^{4}=4 \int Q^{2}$, we get

$$
\begin{aligned}
\frac{3 \int|\nabla h|^{2}}{2 \int|\nabla Q|^{2}}+\frac{\int|h|^{2}}{2 \int Q^{2}} & -\frac{6 \int Q^{2} h_{1}^{2}}{\int Q^{4}} \\
& -\frac{2 \int Q^{2} h_{2}^{2}}{\int Q^{4}}-\frac{\left(\int Q h_{1}\right)^{2}}{2\left(\int Q^{2}\right)^{2}}=\frac{\Phi(h)}{\int Q^{2}}-\frac{\left(\int Q h_{1}\right)^{2}}{2\left(\int Q^{2}\right)^{2}} \geq 0,
\end{aligned}
$$

which implies (A.2).

Step 2. Coercivity. We show that if $h$ fulfils (2.16) and (2.17), then for some $c_{*}$,

$$
\Phi(h) \geq c_{*}\|h\|_{H^{1}}^{2} .
$$


Note that $\Phi(h)=\Phi_{1}\left(h_{1}\right)+\Phi_{2}\left(h_{2}\right)$, where

$$
\begin{aligned}
\Phi_{1}\left(h_{1}\right) & :=\frac{1}{2} \int\left|\nabla h_{1}\right|^{2}+\frac{1}{2} \int h_{1}^{2}-\frac{3}{2} \int Q^{2} h_{1}^{2}=\frac{1}{2} \int\left(L_{+} h_{1}\right) h_{1}, \\
\Phi_{2}\left(h_{2}\right) & :=\frac{1}{2} \int\left|\nabla h_{2}\right|^{2}+\frac{1}{2} \int h_{2}^{2}-\frac{1}{2} \int Q^{2} h_{2}^{2}=\frac{1}{2} \int\left(L_{-} h_{2}\right) h_{2} .
\end{aligned}
$$

By Step $1, L_{+}$is nonnegative on $\{\Delta Q\}^{\perp}$ and $L_{-}$is nonnegative. We will deduce (A.4) from Remark 2.5 and a classical argument (see the proof of Proposition 2.9 in [33]).

We claim that under assumptions (2.16) and (2.17), there exists $c>0$ such that $\Phi_{1}\left(h_{1}\right) \geq c\left\|h_{1}\right\|_{H^{1}}^{2}$. For this we first show that there exists $c_{1}>0$ such that

$$
\begin{aligned}
\int \partial_{x_{1}} Q h_{1} & =\int \partial_{x_{2}} Q h_{1} \\
& =\int \partial_{x_{3}} Q h_{1}=\int \Delta Q h_{1}=0 \Longrightarrow \Phi_{1}\left(h_{1}\right) \geq c_{1}\left\|h_{1}\right\|_{2}^{2} .
\end{aligned}
$$

Following the proof of [33, Proposition 2.9], assume that (A.5) does not hold. Then there exists a sequence of real-valued $H^{1}$-functions $\left\{f_{n}\right\}_{n}$ such that

$$
\begin{gathered}
\lim _{n \rightarrow+\infty} \Phi_{1}\left(f_{n}\right)=0, \quad\left\|f_{n}\right\|_{2}=1 \quad \text { and } \\
\int \Delta Q f_{n}=\int\left(\partial_{x_{1}} Q\right) f_{n}=\int\left(\partial_{x_{2}} Q\right) f_{n}=\int\left(\partial_{x_{3}} Q\right) f_{n}=0 .
\end{gathered}
$$

In particular,

$$
\text { (A.8) } \frac{1}{2} \int\left|\nabla f_{n}\right|^{2}=-\frac{1}{2} \int\left|f_{n}\right|^{2}+\frac{3}{2} \int Q^{2} f_{n}^{2}+o(1)=-\frac{1}{2}+\frac{3}{2} \int Q^{2} f_{n}^{2}+o(1) \text {. }
$$

Thus, $\int\left|\nabla f_{n}\right|^{2} \leq C\left\|f_{n}\right\|_{2}=C$, and hence, $\left\{f_{n}\right\}_{n}$ is bounded in $H^{1}$. Extracting, if necessary, a subsequence from $\left\{f_{n}\right\}_{n}$, we get that there exists $f_{*} \in H^{1}$ such that

$$
f_{n} \underset{n \rightarrow+\infty}{\longrightarrow} f_{*} \text { weakly in } H^{1} .
$$

Since $Q$ is decreasing at infinity, we have

$$
\frac{3}{2} \int Q^{2} f_{n}^{2} \underset{n \rightarrow+\infty}{\longrightarrow} \frac{3}{2} \int Q^{2} f_{*}^{2} .
$$

By (A.8), it follows that

$$
\int Q^{2} f_{*}^{2} \geq \frac{1}{3}
$$


and, in particular, $f_{*} \neq 0$. Furthermore, $\lim \sup _{n}\left\|f_{n}\right\|_{H^{1}} \leq\left\|f_{*}\right\|_{H^{1}}$, and thus, $\lim \sup _{n} \Phi_{1}\left(f_{*}\right) \leq \lim \sup _{n} \Phi_{1}\left(f_{n}\right)$. By (A.6), $\lim \sup _{n} \Phi_{1}\left(f_{*}\right) \leq 0$. By the weak convergence, $\int \Delta Q f_{*}=\int\left(\partial_{x_{1}} Q\right) f_{*}=\int\left(\partial_{x_{2}} Q\right) f_{*}=\int\left(\partial_{x_{3}} Q\right) f_{*}=0$. In particular, by Step $1, \Phi_{1}\left(f_{*}\right) \geq 0$. Therefore,

$$
\frac{1}{2} \int\left(L_{+} f_{*}\right) f_{*}=\Phi_{1}\left(f_{*}\right)=0,
$$

and $f_{*}$ is the solution to the following minimization problem

$$
\begin{aligned}
& 0=\frac{\int\left(L_{+} f_{*}\right) f_{*}}{\left\|f_{*}\right\|_{2}}=\min _{f \in E \backslash\{0\}} \frac{\int\left(L_{+} f\right) f}{\|f\|_{2}}, \quad f_{*} \in E, \text { where } \\
& E:=\left\{f \in H^{1}, \int \Delta Q f=\int\left(\partial_{x_{1}} Q\right) f=\int\left(\partial_{x_{2}} Q\right) f=\int\left(\partial_{x_{3}} Q\right) f=0\right\} .
\end{aligned}
$$

Hence, for some Lagrange multipliers $\lambda_{0}, \ldots, \lambda_{3}$, we can write

$$
L_{+} f_{*}=\lambda_{0} \Delta Q+\lambda_{1} \partial_{x_{1}} Q+\lambda_{2} \partial_{x_{2}} Q+\lambda_{3} \partial_{x_{3}} Q .
$$

By the symmetry of $Q$,

$$
\int\left(\partial_{x_{i}} Q\right) \partial_{x_{j}} Q=0, i \neq j, \quad \int(\Delta Q) \partial_{x_{i}} Q=0
$$

By Remark 2.5, we have $L_{+} \partial_{x_{i}} Q=0$ for $i \in\{1,2,3\}$, which from (A.13) gives

$$
0=-\int f_{*} L_{+}\left(\partial_{x_{i}} Q\right)=\int\left(L_{+} f_{*}\right) \partial_{x_{i}} Q=\lambda_{i} \int\left|\partial_{x_{i}} Q\right|^{2},
$$

showing that $\lambda_{1}=\lambda_{2}=\lambda_{3}=0$. Hence,

$$
L_{+} f_{*}=\lambda_{0} \Delta Q=\lambda_{0}\left(-Q^{3}+Q\right) .
$$

Denote $\widetilde{Q}=Q+x \cdot \nabla Q$. Let us show that

$$
L_{+}\left(\frac{\lambda_{0}}{2}(Q-\widetilde{Q})\right)=\lambda_{0}\left(-Q^{3}+Q\right)=L_{+} f_{*}
$$

Indeed, $L_{+} Q=-2 Q^{3}$. Furthermore, if $Q_{\lambda}(x)=\lambda Q(\lambda x)$, then $\widetilde{Q}:=$ $\frac{\partial}{\partial \lambda}\left(Q_{\lambda}\right)_{\lceil\lambda=1}$. Differentiating the equality $-\lambda^{2} Q_{\lambda}+\Delta Q_{\lambda}+Q_{\lambda}^{3}=0$ with respect to $\lambda$ at $\lambda=1$, we obtain $L_{+} \widetilde{Q}=-2 Q$, which produces (A.15).

By Remark 2.5 and (A.15), there exist $\mu_{1}, \mu_{2}, \mu_{3}$ such that

$$
f_{*}=\frac{\lambda_{0}}{2}(Q-\widetilde{Q})+\mu_{1} \partial_{x_{1}} Q+\mu_{2} \partial_{x_{2}} Q+\mu_{3} \partial_{x_{3}} Q
$$


Next, note that $\int \widetilde{Q} \partial_{x_{j}} Q=0$ (indeed, $\int Q \partial_{x_{j}} Q=0$ by integration by parts, and $\int x_{i} \partial_{x_{i}} Q \partial_{x_{j}} Q=0$ by the symmetry of $\left.Q\right)$. Using that $\int f_{*} \partial_{x_{j}} Q=0$, we get $\mu_{1}=\mu_{2}=\mu_{3}=0$. Hence,

$$
f_{*}=\frac{\lambda_{0}}{2}(Q-\widetilde{Q})=-\frac{\lambda_{0}}{2} x \cdot \nabla Q .
$$

By straightforward calculation, (A.14) and (A.16), we obtain

$$
\Phi_{1}\left(f_{*}\right)=\frac{1}{2} \int\left(L_{+} f_{*}\right) f_{*}=-\frac{\lambda_{0}^{2}}{4} \int \Delta Q(x \cdot \nabla Q)=-\frac{\lambda_{0}^{2}}{8} \int|\nabla Q|^{2} .
$$

By (A.12), $\lambda_{0}=0$, and therefore, $f_{*}=0$, which contradicts (A.11) and concludes the proof of (A.5).

By the explicit expression of $\Phi_{1}$, we have that for $\varepsilon>0$ small enough, $\varepsilon \Phi_{1}\left(h_{1}\right) \geq \frac{\varepsilon}{2} \int\left|\nabla h_{1}\right|^{2}-\frac{c_{1}}{2} \int h_{1}^{2}$ for any $h_{1} \in H^{1}$, where $c_{1}$ is the constant in (A.5). Adding to (A.5), we get that for some constant $c>0$,

$$
\int \partial_{x_{1}} Q h_{1}=\int \partial_{x_{2}} Q h_{1}=\int \partial_{x_{3}} Q h_{1}=\int \Delta Q h_{1}=0 \Longrightarrow \Phi_{1}\left(h_{1}\right) \geq c\left\|h_{1}\right\|_{H^{1}}^{2} .
$$

To conclude the proof of Proposition 2.7, it remains to show that for some constant $c>0$,

$$
\int Q h_{2}=0 \Longrightarrow \Phi_{2}\left(h_{2}\right) \geq c\left\|h_{2}\right\|_{H^{1}}^{2}
$$

The proof is similar to the previous and we omit it.

\section{A.2. Coercivity of $\Phi$ on $G_{\perp}^{\prime}$}

We first show

$$
\forall h \in G_{\perp}^{\prime} \backslash\{0\}, \quad \Phi(h)>0 .
$$

If not, there exists $\tilde{h} \in H^{1} \backslash\{0\}$ such that

$$
\begin{aligned}
\int\left(\partial_{x_{j}} Q\right) \tilde{h}_{1} & =0, j=1,2,3 \\
\int Q \tilde{h}_{2} & =\int \mathcal{Y}_{1} \tilde{h}_{2}=\int \mathcal{Y}_{2} \tilde{h}_{1}=0 \quad \text { and } \quad \Phi(\tilde{h}) \leq 0 .
\end{aligned}
$$

Recall that by Remark 2.5,

$$
\forall h \in H^{1}, \quad B\left(\partial_{x_{1}} Q, h\right)=B\left(\partial_{x_{2}} Q, h\right)=B\left(\partial_{x_{3}} Q, h\right)=B(i Q, h)=0 .
$$


Furthermore, by (A.18),

$$
\begin{aligned}
B\left(\mathcal{Y}_{+}, \tilde{h}\right)=\frac{1}{2} \int\left(L_{+} \mathcal{Y}_{1}\right) \tilde{h}_{1}+ & \frac{1}{2} \int\left(L_{-} \mathcal{Y}_{2}\right) \tilde{h}_{2} \\
& =\frac{1}{2} e_{0} \int \mathcal{Y}_{2} \tilde{h}_{1}-\frac{1}{2} e_{0} \int \mathcal{Y}_{1} \tilde{h}_{2}=0
\end{aligned}
$$

so we have that $\partial_{x_{j}} Q, j=1,2,3, i Q, \mathcal{Y}_{+}$and $\tilde{h}$ are orthogonal in the bilinear symmetric form $B$. Noting that $\Phi(i Q)=\Phi\left(\partial_{x_{j}} Q\right)=\Phi\left(\mathcal{Y}_{+}\right)=0$ and that $\Phi(\tilde{h}) \leq 0$, we get

$$
\forall h \in E:=\operatorname{span}\left\{\partial_{x_{1}} Q, \partial_{x_{2}} Q, \partial_{x_{3}} Q, i Q, \mathcal{Y}_{+}, \tilde{h}\right\}, \quad \Phi(h) \leq 0
$$

We claim that $\operatorname{dim}_{\mathbb{R}} E=6$.

Assume that for some real numbers $\alpha_{j}, \beta, \gamma, \delta$, we have

$$
\sum_{j=1}^{3} \alpha_{j} \partial_{x_{j}} Q+\beta i Q+\gamma \mathcal{Y}_{+}+\delta \tilde{h}=0
$$

By Remark 2.6, $B\left(\mathcal{Y}_{+}, \mathcal{Y}_{-}\right)=-e_{0}\left(L_{-} \mathcal{Y}_{2}, \mathcal{Y}_{2}\right) \neq 0$. Furthermore, the same computation as in (A.19) shows that $B\left(\tilde{h}, \mathcal{Y}_{-}\right)=0$. From (A.20) we get that $\gamma B\left(\mathcal{Y}_{+}, \mathcal{Y}_{-}\right)=0$, which implies $\gamma=0$. Since $\partial_{x_{j}} Q, i Q$ and $\tilde{h}$ are orthogonal in $L^{2}$, we also get that $\alpha_{j}=\beta=\delta=0$. Thus, $\operatorname{dim} E=6$.

We know that $\Phi$ is definite positive on $G_{\perp}$ (a subspace of codimension 5 of $H^{1}$ ), hence, cannot be non-positive on $E$, yielding a contradiction. The proof of (A.17) is complete.

It remains to show that if $h \in G_{\perp}^{\prime}, \Phi(h) \geq c\|h\|_{H^{1}}^{2}$. Let us sketch the proof. As before, it is sufficient to show

$$
\exists c>0, \forall h \in G_{\perp}^{\prime}, \quad \Phi(h) \geq c\|h\|_{2}^{2} .
$$

If not, there exists a sequence $h_{n} \in G_{\perp}^{\prime}$ such that

$$
\lim _{n \rightarrow+\infty} \Phi\left(h_{n}\right)=0, \text { and } \forall n,\left\|h_{n}\right\|_{2}^{2}=1
$$

Extracting a subsequence from $\left(h_{n}\right)$ if necessary, we may assume $h_{n} \rightarrow h^{*}$ weakly in $H^{1}$. The weak convergence of $h_{n}$ implies $h^{*} \in G_{\perp}^{\prime}$. By (A.22) it is easy to check that $h^{*} \neq 0$ and $\Phi\left(h^{*}\right)=0$, which contradicts (A.17), showing as announced (A.21). 


\section{B. Proof of a Cauchy-Schwarz type inequality}

Let us prove Claim 5.4. Let $\mathrm{d}(f)=\int|\nabla Q|^{2}-\int|\nabla f|^{2}$ (so that $\left.\delta(f)=|\mathrm{d}(f)|\right)$ and $\lambda \in \mathbb{R}$. Then $\left\|e^{i \lambda \varphi} f\right\|_{2}=\|Q\|_{2}$. By the Gagliardo-Nirenberg inequality

$$
\left\|\nabla\left(e^{i \lambda \varphi} f\right)\right\|_{2}^{3}\|Q\|_{4}^{4} \geq\|\nabla Q\|_{2}^{3}\|f\|_{4}^{4} .
$$

Raising the previous inequality to the power $\frac{2}{3}$, and expanding $\left\|\nabla\left(e^{i \lambda \varphi} f\right)\right\|_{2}^{2}$, we get

$$
\lambda^{2} \int|\nabla \varphi|^{2}|\nabla f|^{2}+2 \lambda \operatorname{Im} \int(\nabla \varphi \cdot \nabla f) \bar{f}+\int|\nabla f|^{2}-\|f\|_{4}^{\frac{8}{3}} \frac{\|\nabla Q\|_{2}^{2}}{\|Q\|_{4}^{\frac{8}{3}}} \geq 0 .
$$

Using elementary properties of quadratic inequalities (in $\lambda$ ), we obtain

$$
\left|\operatorname{Im} \int(\nabla \varphi \cdot \nabla f) \bar{f}\right|^{2} \leq\left(\int|\nabla \varphi|^{2}|\nabla f|^{2}\right)\left(\int|\nabla f|^{2}-\|f\|_{4}^{\frac{8}{3}} \frac{\|\nabla Q\|_{2}^{2}}{\|Q\|_{4}^{\frac{8}{3}}}\right) .
$$

We have

$$
\|\nabla f\|_{2}^{2}=\|\nabla Q\|_{2}^{2}-\mathrm{d}(f)
$$

and, by assumption (5.12), we have

$$
\|f\|_{4}^{4}=\|Q\|_{4}^{4}-2 \mathrm{~d}(f),
$$

so that

$$
\begin{array}{r}
\int|\nabla f|_{2}^{2}-\|f\|_{4}^{\frac{8}{3}} \frac{\|\nabla Q\|_{2}^{2}}{\|Q\|_{4}^{\frac{8}{3}}}=\|\nabla Q\|_{2}^{2}-\mathrm{d}(f)-\left(\|Q\|_{4}^{4}-2 \mathrm{~d}(f)\right)^{\frac{2}{3}} \frac{\|\nabla Q\|_{2}^{2}}{\|Q\|_{4}^{\frac{8}{3}}} \\
=\|\nabla Q\|_{2}^{2}-\mathrm{d}(f)-\|\nabla Q\|_{2}^{2}+\frac{4}{3} \frac{\mathrm{d}(f)}{\|Q\|_{4}^{4}}\|\nabla Q\|_{2}^{2}+O\left(\left|\mathrm{~d}^{2}(f)\right|\right) .
\end{array}
$$

Recalling that $\|Q\|_{4}^{4}=\frac{4}{3}\|\nabla Q\|_{2}^{2}$, we obtain

$$
\int|\nabla f|_{2}^{2}-\|f\|_{4}^{\frac{8}{3}} \frac{\|\nabla Q\|_{2}^{2}}{\|Q\|_{4}^{\frac{8}{3}}}=O\left(\left|\mathrm{~d}^{2}(f)\right|\right),
$$

concluding the proof of the claim.

\section{References}

[1] Bahouri, H. ANd GÉRARd, P.: Concentration effects in critical nonlinear wave equation and scattering theory. In Geometrical optics and related topics (Cortona, 1996), 17-30. Progr. Nonlinear Differential Equations Appl. 32. Birkhäuser Boston, Boston, MA, 1997. 
[2] BANicA, V.: Remarks on the blow-up for the Schrödinger equation with critical mass on a plane domain. Ann. Sc. Norm. Super. Pisa Cl. Sci. (5) 3 (2004), no. 1, 139-170.

[3] Bourgain, J.: Global wellposedness of defocusing critical nonlinear Schrödinger equation in the radial case. J. Amer. Math. Soc. 12 (1999), no. $1,145-171$.

[4] Cazenave, T.: Semilinear Schrödinger equations. Courant Lecture Notes in Mathematics 10. New York University, Courant Institute of Mathematical Sciences, New York. AMS, Providence, RI, 2003.

[5] Coffman, C.V.: Uniqueness of the ground state solution for $\Delta u-u+u^{3}=0$ and a variational characterization of other solutions. Arch. Rational Mech. Anal. 46 (1972), 81-95.

[6] Colliander, J., Keel, M., Staffilani, G., Takaoka, H. and Tao, T.: Global existence and scattering for rough solutions of a nonlinear Schrödinger equation on $\mathbb{R}^{3}$. Comm. Pure Appl. Math. 57 (2004), no. 8, 987-1014.

[7] Duyckaerts, T., Holmer, J. and Roudenko, S.: Scattering for the non-radial 3D cubic nonlinear Schrödinger equation. Math. Res. Lett. 15 (2008), no. 6, 1233-1250.

[8] Duyckaerts, T. And Merle, F.: Dynamic of threshold solutions for energy-critical NLS. To appear in Geom. Funct. Anal., 2007.

[9] Duyckaerts, T. and Merle, F.: Dynamics of threshold solutions for energy-critical wave equation. Int. Math. Res. Pap. IMRP 2007, no. 4, Art. ID rpn002, 67 pp. (2008).

[10] GÉrard, P.: Description du défaut de compacité de l'injection de Sobolev. ESAIM Control Optim. Calc. Var. 3 (1998), 213-233 (electronic).

[11] GÉrard, P.: A critical profile decomposition for the three-dimensional cubic wave equation. Manuscript in preparation.

[12] Ginibre, J. and Velo, G.: On a class of nonlinear Schrödinger equations. I. The Cauchy problem, general case; II. Scattering theory, general case. J. Funct. Anal. 32 (1979), no. 1, 1-32, 33-71.

[13] Grillakis, M.: Analysis of the linearization around a critical point of an infinite-dimensional Hamiltonian system. Comm. Pure Appl. Math. 43 (1990), no. 3, 299-333.

[14] Holmer, J. And Roudenko, S.: On blow-up solutions to the 3D cubic nonlinear Schrödinger equation. Appl. Math. Res. Express AMRX 2007, no. 1, Art. ID abm004, 31pp.

[15] Holmer, J. And Roudenko, S.: A sharp condition for scattering of the radial 3D cubic nonlinear Schrödinger equation. Comm. Math. Phys. 282 (2008), no. 2, 435-467.

[16] Kenig, C. E. And Merle, F.: Global well-posedness, scattering and blowup for the energy-critical, focusing, non-linear Schrödinger equation in the radial case. Invent. Math. 166 (2006), no. 3, 645-675. 
[17] Kenig, C. E. And Merle, F.: Global well-posedness, scattering and blowup for the energy-critical focusing non-linear wave equation in the radial case. Acta Math. 201 (2008), no. 2, 147-212.

[18] Kenig, C. E. And Merle, F.: Scattering for $\dot{H}^{1 / 2}$ bounded solutions to the cubic defocusing NLS in 3D. To appear in Trans. Amer. Math. Soc.

[19] Keranin, S.: On the defect of compactness for the Strichartz estimates of the Schrödinger equations. J. Differential Equations 175 (2001), no. 2, 353-392.

[20] Killip, R., TaO, T. and Visan, M.: The cubic nonlinear Schrödinger equation in two dimensions with radial data. To appear in J. Eur. Math. Soc.

[21] Killip, R. And Visan, M.: The focusing energy-critical nonlinear Schrödinger equation in dimensions five and higher. To appear in Amer. J. Math.

[22] Killip, R., Visan, M. and Zhang, X.: The mass-critical nonlinear Schrödinger equation with radial data in dimensions three and higher. Anal. PDE 1 (2008), no. 2, 229-266.

[23] Kwong, M. K.: Uniqueness of positive solutions of $\Delta u-u+u^{p}=0$ in $\mathbb{R}^{n}$. Arch. Rational Mech. Anal. 105 (1989), no. 3, 243-266.

[24] Lions, P.-L.: The concentration-compactness principle in the calculus of variations. The locally compact case. II. Ann. Inst. H. Poincaré Anal. Non Linéaire 1 (1984), no. 4, 223-283.

[25] Lions, P.-L.: The concentration-compactness principle in the calculus of variations. The limit case. II. Rev. Mat. Iberoamericana 1 (1985), no. 2, $45-121$.

[26] Merle, F.: On uniqueness and continuation properties after blow-up time of self-similar solutions of nonlinear Schrödinger equation with critical exponent and critical mass. Comm. Pure Appl. Math. 45 (1992), no. 2, 203-254.

[27] Merle, F.: Determination of blow-up solutions with minimal mass for nonlinear Schrödinger equations with critical power. Duke Math. J. 69 (1993), no. $2,427-454$.

[28] Merle, F. And Raphä̈L, P.: Blow up of the critical norm for some radial $L^{2}$ super critical nonlinear Schrödinger equations Amer. J. Math. 130 (2008), no. 4, 945-978.

[29] Merle, F. And Vega, L.: Compactness at blow-up time for $L^{2}$ solutions of the critical nonlinear Schrödinger equation in 2D. Internat. Math. Res. Notices 1998, no. 8, 399-425.

[30] Strauss, W. A.: Existence of solitary waves in higher dimensions. Comm. Math. Phys. 55 (1977), no. 2, 149-162.

[31] TAO, T.: Nonlinear dispersive equations. Local and global analysis. CBMS Regional Conference Series in Mathematics 106. Published for the Conference Board of the Mathematical Sciences, Washington, DC; by the American Mathematical Society, Providence, RI, 2006. 
[32] Weinstein, M. I.: Nonlinear Schrödinger equations and sharp interpolation estimates. Comm. Math. Phys. 87 (1982/83), no. 4, 567-576.

[33] Weinstein, M. I.: Modulational stability of ground states of nonlinear Schrödinger equations. SIAM J. Math. Anal. 16 (1985), no. 3, 472-491.

[34] Weinstein, M. I.: Lyapunov stability of ground states of nonlinear dispersive evolution equations. Comm. Pure Appl. Math. 39 (1986), no. 1, $51-67$.

Recibido: 9 de julio de 2008

Thomas Duyckaerts

Université de Cergy-Pontoise UMR CNRS 8088, France thomas . duyckaerts@u-cergy.fr

Svetlana Roudenko Arizona State University

Tempe AZ 85287, USA svetlana@math.asu.edu

T.D. was partially supported by the French ANR Grant ONDNONLIN. Part of this work was done during S.R. visit to the University of Cergy-Pontoise funded by the Grant ONDNONLIN. Both authors would like to thank Frank Merle for fruitful discussions on the subject and Vianney Combet for careful reading of an earlier version of the paper and remarks improving its content. 